\title{
ON SUCCESSIVE APPROXIMATIONS IN HOMOLOGICAL ALGEBRA
}

\author{
BY \\ V. K. A. M. GUGENHEIM AND R. J. MILGRAM
}

A major problem in homological algebra is the effective calculation of $\operatorname{Ext}_{A}(K, K)$ for a graded algebra over a field $K$. In topology, the problem comes up in evaluating the $E_{2}$-term of the Eilenberg-Moore spectral sequence and the $E_{2}$-term of the Adams spectral sequence.

In these and many other cases the algebra $A$ is a coassociative cocommutative Hopf algebra. By the (dual) Borel theorem (at least if $K$ is perfect) such an algebra is centrally solvable in the sense that there is a sequence of Hopf algebras and maps

$$
A \rightarrow \cdots \rightarrow \underset{\uparrow}{\substack{Q_{n} \\ A_{n}}} \underset{\substack{\uparrow \\ A_{2}}}{A_{2}} \rightarrow Q_{1}=1
$$

so that $Q_{i}=Q_{i+1} / / A_{i+1}$ and $A=$ proj lim $Q_{i}$; and each $A_{i}$ is commutative.

Consequently, as a $K$-module $A$ is isomorphic to $\bigotimes_{i} A_{i}$, but the multiplication $\Phi$ differs from the tensor-product multiplication $\Phi_{0} . \Phi$ and $\Phi_{0}$, however, are closely related. This idea was explored in [1] and [2].

In this paper we take suitable resolutions $U_{i} \otimes A_{i}$ (e.g. the bar-construction $\left.B\left(A_{i}\right)\right)$, form $U \otimes A=\left(\bigotimes_{i} U_{i}\right) \otimes A \approx \bigotimes_{i}\left(U_{i} \otimes A_{i}\right)$, start from the tensor-product differential $d$ appropriate to $\Phi_{0}$ and then form successive "approximate differentials" $D_{1}, D_{2}, D_{3}, \ldots$ by a perturbation process which, in the limit, gives a differential $D$ appropriate to $\Phi$. Since $\bigotimes_{i}\left(U_{i} \otimes A_{i}\right)$ is usually much smaller than-saythe bar-construction for $A$, this gives us a small resolution and hence effective methods of computation.

The essential property of the resolutions $U_{i} \otimes A_{i}$ is that they should be DGAalgebras; since $A_{i}$ is commutative, $B\left(A_{i}\right)$ satisfies this condition. If $A_{i}$ is written as a product of monogenics $A_{i 1} \otimes A_{i 2} \otimes \cdots$ and $K$ has characteristic $p, A_{i, j}$ is a polynomial algebra on a generator $e_{i, j}$ truncated by $\left(e_{i,}\right)^{p_{k}}=0$ for some $k$. For these cases, the bar-construction has a simple form; thus, if $A_{i, j}$ is an exterior algebra, $B\left(A_{i, j}\right)$ is a divided polynomial algebra given by $e_{r} e_{s}=(r, s) e_{r+s}$ if $e_{r}$ $=\left[e_{i, j}, \ldots, e_{i, j}\right]$ ( $r$ times). Thus, in this case, we get quite small, indeed minimal, resolutions.

Received by the editors March 4, 1969. 
In particular, the Steenrod algebra $\mathscr{A}(2)$ can be written as a twisted tensorproduct of exterior algebras on generators $e_{i, j}$ of degree $2^{j}\left(2^{i}-1\right)$. Thus, for calculating $\operatorname{Ext}_{\mathscr{A}(2)}\left(Z_{2}, Z_{2}\right)$ we obtain a resolution which looks like a polynomial algebra on generators $h_{i j}$ of bidegree $\left(1,2^{j}\left(2^{i}-1\right)\right)$; the dual of a divided polynomial algebra being a polynomial algebra.

In the case where truncated polynomial generators of higher truncation occur, the associated bar-constructions are no longer minimal-but they are still quite manageable. J. P. May, in his thesis, tries to obtain resolutions for his "associated graded algebras" by using tensor-products of Koszul resolutions which are smaller than the bar-constructions; but difficulties result precisely because these resolutions are not DGA-algebras.

The basic idea of our sequence of differentials $D_{1}, D_{2}, \ldots$ is the following: Each $D_{n}: U \otimes A \rightarrow U \otimes A$ is a map of $(A, \Phi)$-modules; but $D_{n}^{2} \neq 0$. The difference between $\Phi$ and $\Phi_{0}$ is best expressed in terms of a certain filtration $\left\{F_{p}\right\}$ of $U \otimes A$ and $D_{n}^{2} F_{p} \subset F_{p-n-2}$. This leads to convergence.

Similarly, we can start with a map which is a chain-map for the (untwisted) $d$; and obtain a convergent sequence of maps the limit of which is a chain-map for $D$.

The filtration used in all this is, of course, related to a spectral sequence. One might say that a filtration can be used in two ways: Analytically, to investigate a given situation, or synthetically, to construct new differentials, maps, homotopies, etc. The former is achieved, classically, by the method of spectral sequences; the latter is the essence of the present paper.

The filtration we use for this is especially adapted to the process in hand; it is not, in particular, the "augmentation filtration" used so successfully by J. P. May. Under certain circumstances, however, the augmentation filtration can be closely related to our method; the conditions for this are fulfilled, for instance, in the case of the Steenrod algebra. In such cases, the combination of our (synthetic) methods with the (analytic) use of the "May spectral sequence" appears to give a powerful tool for computation; we try to illustrate this by the calculations which begin in $\S 5$. This same case has been treated, using different methods by Mahowald and Copeland, who have set up a machine computation, and by N. Shimada.

We are much indebted to many conversations with J. P. May; and to A. Liulevicius whose careful reading of our paper has eliminated many obscurities, and some errors.

0 . Preliminary definitions. $K$ is a commutative ring with unit $1 \in K$. Module $X=\left\{X_{n}\right\}$ ( $n \in$ the integers) will mean "graded module over $K$ "; i.e., a sequence of modules $X_{n}$ in the ungraded sense. If $X_{n}=0$ for $n<0$, we call $X$ a positive module. We identify $K$ with the graded module $\left\{K_{n}\right\}$ where $K_{0}=K, K_{n}=0$ for $n \neq 0$.

If $X, Y$ are modules $X \otimes Y$ is defined as the module with

$$
(X \otimes Y)_{n}=\sum_{p+q=n} X_{p} \otimes Y_{q}
$$


$\operatorname{Hom}(X, Y)$ by $\operatorname{Hom}_{n}(X, Y)=(\operatorname{Hom}(X, Y))_{n}=\left\{\operatorname{Hom}_{K}\left(X_{p}, Y_{p+n}\right)\right\}$. We embed $\operatorname{Hom}(X, Y) \otimes \operatorname{Hom}\left(X^{\prime}, Y^{\prime}\right)$ in Hom $\left(X \otimes X^{\prime}, Y \otimes Y^{\prime}\right)$ by the definition $\left(f \otimes f^{\prime}\right)$ $\cdot\left(x \otimes x^{\prime}\right)=(-1)^{p^{\prime} q} f x \otimes f^{\prime} x^{\prime}$ if $x \in X_{q}, f^{\prime} \in \operatorname{Hom}_{p^{\prime}}\left(X^{\prime}, Y^{\prime}\right)$. If $f, g \in \operatorname{Hom}(X, X)$ have gradings $p, q$ respectively we define the commutator $[f, g]=f g-(-1)^{p q} g f$. A connected module $X$ will be a positive module with $X_{0}=K$; in this case the unit and augmentation $\eta: K \rightarrow X, \varepsilon: X \rightarrow K$ are defined in the evident way; a map of connected modules $f: X \rightarrow Y$ is an element of $\operatorname{Hom}_{0}(X, Y)$ such that $f \mid X_{0}=$ the identity.

Let $X_{i}(i=1,2, \ldots)$ be a sequence of connected modules (the $n$-dimensional component of $X_{i}$ is $X_{i, n}$ ); we embed $X_{1} \otimes \cdots \otimes X_{i}$ in $X_{1} \otimes \cdots \otimes X_{i+1}$ in the evident way and define

$$
\otimes X_{i}=\bigotimes_{i=1}^{\infty} X_{i}=\bigcup_{i=1}^{\infty} X_{1} \otimes \cdots \otimes X_{i}
$$

An algebra $A=(A, \varphi)$ will be a connected module together with an element $\varphi \in \mathrm{Hom}_{0}(A \otimes A, A)$ satisfying the usual conditions (including associativity). $A$ (or, rather, $\varphi$ ) will be said to be commutative if $\varphi(x \otimes y)=(-1)^{p q} \varphi(y \otimes x)$ for $x \in A_{p}, y \in A_{q}$.

The meaning of terms like "map of algebras", " $A$-module", "map of $A$ modules", etc., are the usual ones.

For a connected module $X, I(X)$ denotes the "augmentation ideal", i.e.,

$$
(I(X))_{0}=0, \quad(I(X))_{n}=X_{n} \text { if } n>0 .
$$

In the sequel much use will be made of bigraded modules $U=\left\{U_{m, n}\right\}$. If $U$ and $V$ are bigraded, we define $U \otimes V$ by

$$
(U \otimes V)_{m, n}=\sum_{p+q=m, r+s=n} U_{p, r} \otimes V_{q, s}
$$

if $X=\left\{X_{n}\right\}$ is graded, bigrade $U \otimes X$ by

$$
(U \otimes X)_{m, n}=\sum_{r+s=n} U_{m, r} \otimes X_{s}
$$

A map of bigraded modules $G: U \rightarrow V$ of type $(k, e)$ is a sequence of maps $U_{m, n} \rightarrow V_{m+k, n+e}$.

We shall always refer to the first grading as the degree, the second as the dimension; thus, the grading of singly graded modules will be regarded as a dimension.

Most of the maps we shall consider will be of type $(k, 0)$; the various formulas for signs then simply reflect the degree; cf. [4, p. 299].

If $U=\left\{U_{m, n}\right\}$ is positive in both gradings, we define $I U$ by

$$
\begin{aligned}
(I U)_{m, n} & =U_{m, n} & & \text { if } m>0 \text { or } n>0, \\
& =0 & & \text { if } m=n=0 .
\end{aligned}
$$

The projection $U \rightarrow U_{0,0} \subset U$ will be denoted generically by $\Pi_{0}$. 


\section{Twisted algebras.}

1.0. Let $\left(A_{i}, \varphi_{i}\right)(i=1,2, \ldots)$ be a sequence of algebras and $A=\bigotimes A_{i}$. We can give $A$ a product $\Phi_{0}: A \otimes A \rightarrow A$ in the usual way: Suppose $a_{i} \in A_{i, n_{i}}, b_{i} \in A_{i, m_{i}}$ and $a=a_{1} \otimes \cdots \otimes a_{i}, b=b_{1} \otimes \cdots \otimes b_{i} ;$ then

$$
\begin{aligned}
\Phi_{0}(a \otimes b) & =a b, \\
& =(-1)^{8} a_{1} b_{1} \otimes \cdots \otimes a_{i} b_{i},
\end{aligned}
$$

where $\varepsilon=m_{1}\left(n_{2}+\cdots+n_{i}\right)+m_{2}\left(n_{3}+\cdots+n_{i}\right)+\cdots+m_{i-1} n_{i}$ and $a_{i} b_{i}=\varphi_{i}\left(a_{i} \otimes b_{i}\right)$. Using this notation and the embedding implicit in the definition of $\otimes A_{i}$, we shall often write $a_{1} a_{2} \cdots a_{i}=a_{1} \otimes \cdots \otimes a_{i}$; etc.

Into this context we introduce the following "weighted dimension filtration": $F_{p}=F_{p} A$ is the submodule generated by terms of the form $a_{1} \otimes a_{2} \otimes \cdots \otimes a_{i}$, where $a_{i} \in A_{i, n_{i}}$ such that

$$
n_{1}+2 n_{2}+\cdots+i n_{i} \geqq-p .
$$

Clearly $F_{p} A=A$ if $p \geqq 0, F_{p} \subset F_{p+1}, \bigcap_{p} F_{p}=0, \Phi_{0}\left(F_{p} \otimes F_{q}\right) \subset F_{p+q}$.

All this being given we make the following

1.1 Definition. $(A, \Phi)$, or more precisely $\left(\left\{A_{i}, \varphi_{i}\right\}, \Phi\right)$ is a twisted algebra if $\Phi: A \otimes A \rightarrow A$ is an associative product with unit and the following conditions are satisfied:

(0) Each $\varphi$ is commutative.

(1) (Convergence Assumption). There is an integer-valued function on $\gamma(i)$ with $\gamma(i) \rightarrow \infty$ as $i \rightarrow \infty$ such that $A_{i, n}=0$ if $0<n<\gamma(i)$.

(2) $\left(\Phi-\Phi_{0}\right)\left(F_{p} \otimes F_{q}\right) \subset F_{p+q-1}$.

(3) $\left(\Phi-\Phi_{0}\right)\left(A \otimes I A_{i}+I A_{i} \otimes A\right) \subset \sum_{j=i}^{\infty} A_{1} \otimes \cdots \otimes A_{j} \otimes I A_{j+1}$.

It follows from (2) that $\Phi\left(F_{p} \otimes F_{q}\right) \subset F_{p+q}$. We shall write $a \circ b=\Phi(a \otimes b)$ and refer to $\Phi, \Phi_{0}$ as the twisted, untwisted multiplication respectively.

Suppose $\left(\left\{B_{i}, \varphi_{i}\right\}, \Phi\right)$ is another twisted algebra, where with the customary abuse of notation we use symbols like $\varphi_{i}, \Phi$ generically, and $g_{i}: A_{i} \rightarrow B_{i}$ a sequence of maps of algebras, then $g=\bigotimes_{i=1}^{\infty} g_{i}: A \rightarrow B$ is called a map of twisted algebras if $\Phi(g \otimes g)=g \Phi$. Thus, for $g$ to be map of twisted algebras we require $g(a \circ b)$ $=g(a) \circ g(b)$ and $g(a b)=g(a) g(b)$.

Note that in all these definitions the case of a finite tensor-product is included: condition $1.1(1)$ is then vacuous.

1.2 EXAMPLES.

1.21. Let $A_{1}, A_{2}$ be algebras. We then have the concept of the "twisted tensorproduct of algebras", cf. [1, 8.1*]. Here we have the conditions

$$
\begin{gathered}
a_{1} \circ a_{2}= \pm a_{2} \circ a_{1}=a_{1} \otimes a_{2}, \quad a_{2} \circ a_{2}^{\prime}=a_{2} a_{2}^{\prime}, \\
a_{1} \circ a_{1}^{\prime}-a_{1} a_{1}^{\prime} \in A_{1} \otimes I\left(A_{2}\right) .
\end{gathered}
$$

Condition 1.1(2) follows easily. 
The iteration of twisted tensor-products of algebras will, in general, not produce a twisted algebra in the present sense. An important exception arises as follows:

1.22. Let $\left(A_{i}, \varphi_{i}, \psi_{i}\right)$ be fully commutative Hopf algebras (i.e., $\varphi_{i}$ and $\psi_{i}: A_{i} \rightarrow$ $A_{i} \otimes A_{i}$ are both commutative), and suppose that $Q_{i}=\left(A_{1} \otimes \cdots \otimes A_{i}, \Phi_{i}, \Psi_{i}\right)$ is a Hopf algebra where we assume inductively (i.e. for each $i$ ): (i) $Q_{i+1}=Q_{i}$ $\otimes A_{i+1}$ is an extension of Hopf algebras in the sense of [1], (ii) the twisting functions $\beta: Q_{i} \rightarrow A_{i+1} \otimes A_{i+1}$ are zero.

What condition (ii) amounts to is that $\Psi_{i}$ is, for each $i$, simply the untwisted coproduct.

In this case we can prove (using the formulas of [1]):

1.221 Lemma. $\left(A_{1} \otimes \cdots \otimes A_{i}, \Phi_{i}\right)$ satisfies condition $1.1(2)$; i.e., is a twisted algebra.

The most important case of this is the representation of the Steenrod algebra given by Milnor: The fact that the coproduct is "untwisted" in this case follows from the fact that the dual of the Steenrod algebra is a polynomial algebra-i.e. has an "untwisted" product in terms of the representation. The fact that the Milnor representation of the Steenrod algebra satisfies 1.1(2) (1.1(0) and 1.1(1) are obvious) can, of course, be read off directly from the multiplication formulas (i.e. the comultiplication formulas of the dual algebra). We leave these details to the reader.

Returning now to the case of 1.22 we see that the untwisted coproduct, when regarded as a map $(A, \Phi) \rightarrow\left(A \otimes A, \Phi_{A \otimes A}\right)$ is precisely a map of twisted algebras in the sense of 1.1 ; here $A \otimes A$ is, of course, regarded as the twisted algebra $\bigotimes_{i}\left(A_{i} \otimes A_{i}\right)$.

\section{Twisted complexes.}

2.0. Let $A$ be an algebra. We shall say that $(U, A, d)$ is a complex (over $A$ ) if $U=\left\{U_{m, n}\right\}$ is a bigraded $K$-module, positive in each grading with $U_{m, 0}=0$ if $m>0$, $U_{0,0}=K$; and $d: U \otimes A \rightarrow U \otimes A$ is a map of $A$-modules, of type $(-1,0)$, such that $d^{2}=0, \Pi_{0} d=0$.

If there exists a map of bimodules $s: U \otimes A \rightarrow U \otimes A$ of type $(1,0)$ such that

$$
d s+s d=[d, s]=\text { identity }-\Pi_{0},
$$

then we say that $(U, A, d, s)$ is an acyclic complex, or a resolution of $A$.

ExAmple. The bar-resolution, cf. p. 299 of [4]. The bar-resolution satisfies the additional conditions:

$$
s(U \otimes A) \subset U, \quad s U=0
$$

which define it uniquely.

If $A$ is a commutative algebra, the bar-resolution $B(A)=\bar{B}(A) \otimes A$ is a commutative DGA-algebra; the product induces in $\bar{B}(A)$ the structure of a commutative algebra, cf. [4, p. 312]. Moreover, by $1.1(1) \bar{B}\left(A_{i}\right)_{m, k}=0$ if $k<m \gamma(i)$. 
Accordingly we make

2.01 Definition. $\left(U_{i}, A_{i}, d_{i}\right)$ is called a good resolution if

(i) $\left(U_{i} \otimes A_{i}, d_{i}\right)$ is a DGA-algebra.

(ii) The product induces in $U_{i}$ the structure of an algebra.

(iii) $U_{i, m, k}=0$ if $k<$ some function of $m$ and $i$ which $\rightarrow \infty$ as $i \rightarrow \infty,(m, k) \neq(0,0)$.

2.1. Let $(A, \Phi)=\left(\left\{A_{i}, \varphi_{i}\right\}, \Phi\right)$ be a twisted algebra. Suppose $\left(U_{i}, A_{i}, d_{i}\right)$ is a good resolution for $A_{i}$, with chain-homotopy $\left(s_{i}\right)$. We define $U=\bigotimes_{i=1}^{\infty} U_{i}=U_{1}$ $\otimes U_{2} \cdots$. We extend the filtration of 1.0 to $U \otimes A$ as follows: $F_{p}=F_{p}(U \otimes A)$ is the submodule generated by all terms of the form

$$
u_{1} \otimes u_{2} \otimes \cdots \otimes u_{i} \otimes a
$$

where $u_{i} \in U_{i, m_{i}, n_{i}}$ such that

$$
n_{1}+2 n_{2}+\cdots+i n_{n}-(\text { filtration } a)-m_{1}-m_{2}-\cdots-m_{i} \geqq-p .
$$

It is well known that a resolution $(U, A, d)$ for the algebra $\left(A, \Phi_{0}\right)$ is obtained if we choose $d=d_{1}+d_{2}+\cdots$, where, with an evident "abuse of notation" we denote by $d_{i}: U \otimes A \rightarrow U \otimes A$ the evident map induced by $d_{i}$; we leave it to the reader to fill in the matter of the appropriate sign $\cdots ; s=t_{1}+t_{2}+\cdots$ where, observing that $U \otimes A \approx\left(U_{1} \otimes A_{1}\right) \otimes\left(U_{2} \otimes A_{2}\right) \otimes \cdots, \quad t_{i}=\left(U_{1} \otimes A_{1}\right) \otimes \cdots$ $\otimes\left(U_{i-1} \otimes A_{i-1}\right) \otimes s_{i} \otimes \varepsilon_{i+2} \otimes \cdots$ and $\varepsilon_{i}$ stands for projection on terms of bigrading $(0,0)$ in $\left(U_{i} \otimes A_{i}\right)$; we use $X$ for the identity map of the object $X$.

It is now easily seen that $d F_{p} \subset F_{p-1}, s F_{p} \subset F_{p+1}$.

Since $\left(U_{i}, A_{i}, d_{i}\right)$ is a good resolution, cf. $2.01, U_{i}$ is an algebra. We can thus give $U$ the structure of an algebra with the usual (untwisted!) formula for tensorproducts. Thus $U \otimes A$ has two algebra structures:

(i) (Untwisted): Using $\Phi_{0}$ for $A$. We shall denote this extend product also by $\Phi_{0}$.

(ii) (Twisted): Using $\Phi$ for $A$. This will still be denoted by $\Phi$.

Note that the two products differ only in regard to $A$ : We have the same product for $U$.

$\left(U, A, d, \Phi_{0}\right)$ is a differential algebra. It is easily seen that

$$
\Phi_{0}\left(F_{p} \otimes F_{q}\right) \subset F_{p+q} .
$$

Also, formula 1.1(2) holds with the extended meanings of $\Phi, F_{p}$ and hence

$$
\Phi\left(F_{p} \otimes F_{q}\right) \subset F_{p+q} .
$$

If $w, w^{\prime} \in U \otimes A$, we shall continue to use the notations $\Phi_{0}\left(w \otimes w^{\prime}\right)=w w^{\prime}$, $\Phi\left(w \otimes w^{\prime}\right)=w \circ w^{\prime}$.

2.2. With all the data of 2.1 , we make the following

Definitions. (i) $\Pi_{i}: U \otimes A \rightarrow U \otimes A$ is the projection onto the summand $\left(U_{1} \otimes A_{1}\right) \otimes \cdots \otimes\left(U_{i-1} \otimes A_{i-1}\right) \otimes I\left(U_{i} \otimes A_{i}\right)$ if $i>0 . \Pi_{0}$ is the projection onto terms of bigrading $(0,0)$. If $\mathrm{I}_{i}$ denotes the identity on $U_{i} \otimes A_{i}$, then $\Pi_{i}=\mathrm{I}_{i} \otimes \cdots$ $\otimes I_{i-1} \otimes\left(I_{i}-\varepsilon_{i}\right) \otimes \varepsilon_{i+1} \otimes \varepsilon_{i+2} \otimes \cdots$. 
(ii) $\iota_{i}: U_{1} \otimes \cdots \otimes U_{i} \rightarrow U \otimes A, \imath^{i+1}: U_{i+1} \otimes U_{i+2} \otimes \cdots \rightarrow U \otimes A, \iota_{A}: A \rightarrow$ $U \otimes A$ are the natural injections.

(iii) If $G: U \otimes A \rightarrow U \otimes A$ is a map of bigraded $K$-modules, we define $L(G): U \otimes A \rightarrow U \otimes A$ by

$$
L(G)=\sum_{i=1}^{\infty} \Phi\left\{\Pi_{i+1} G \iota_{i} \otimes \imath^{i+1} \otimes \iota_{A}\right\}
$$

(we extend the meaning of $\Phi$ to 3 factors!).

A map of $(A, \Phi)$-modules will be said to be $\Phi$-linear. Clearly $L(G)$ is $\Phi$-linear. A much simpler way of deriving from $G$ a $\Phi$-linear map is given by

(iv) $l(G)=\Phi\left(G \iota_{\infty} \otimes \iota_{A}\right)$.

Notice that if $G F_{p} \subset F_{p+k}$ (for all $p$ and a fixed $k$ ), then the same is true of $L(G)$ and $l(G)$.

2.31 Definition. $(U, A, D, d)$ or, less accurately, $(U, A, D)$ will be called a twisted complex over the twisted algebra $A=(A, \Phi)$ if we are in the situation of 2.1, 2.2 and if

(i) $(U, A, D)$ is a complex over $(A, \Phi)$.

(ii) $(D-d) F_{p} \subset F_{p-2}, L(D-l(d))=D-l(d)$.

The twisted complex will be called a twisted resolution if there is a contracting chain-homotopy $S$ such that $S F_{p} \subset F_{p+1}$.

2.32. Next, let $g=\bigotimes g_{i}: A \rightarrow B$ be a map of twisted algebras, cf. 1.1, and let $(V, B, D)$ be a twisted complex over the twisted algebra $B$. Since we have only used good resolutions, cf. 2.01, $U_{i} \otimes A_{i}, V_{i} \otimes B_{i}$ are differential algebras. We now make a further assumption:

2.321 Assumption. (i) $g_{i}$ induces a map of DGA-algebras $U_{i} \otimes A_{i} \rightarrow V_{i} \otimes B_{i}$.

(ii) $g_{i} U_{i} \subset V_{i}$, so that $g_{i} \mid U_{i}: U_{i} \rightarrow V_{i}$ is a map of algebras.

The main examples are: Our "good resolutions" are in fact bar-constructions; or $g_{i}$ is the identity map.

2.322 Notations. The induced map $U_{i} \otimes A_{i} \rightarrow V_{i} \otimes B_{i}$ will still be denoted by $g_{i}$; the map $\otimes g_{i}: U \otimes A \rightarrow V \otimes B$ will still be denoted by $g$. Now, let $G: U \otimes A$ $\rightarrow V \otimes B$ be a map of bigraded $K$-modules, of type $(k, 0)$. We define $L_{g}(G): U \otimes A$ $\rightarrow V \otimes B$, of the same type, by

$$
L_{g}(G)=\sum_{i=1}^{\infty} \Phi\left\{\Pi_{i+1} G \iota_{i} \otimes g^{i+1} \otimes \iota_{B} g\right\}
$$

where $g^{i+1}=\imath^{i+1}\left(g_{i+1} \otimes \cdots\right)$. Clearly $L_{g}(G)$ is " $g$-linear", i.e., $L_{g}(G)(u \otimes a)$ $=L_{g}(G)(u) \circ g(a)$. A much simpler way of obtaining a $g$-linear map is $l_{g}(G)$ $=\Phi\left\{G \iota_{\infty} \otimes g\right\}$. Clearly $L, l$ of 2.2 are special cases of $L_{g}, l_{g}$ for $g=$ identity. Note $l_{g}(g)=g, L_{g}(g)=0$; recall that we are using the symbol $g$ for a map $A \rightarrow B$; and its extension to the resolutions!

In all these formulas we have used, informally, the isomorphism $\otimes_{i}\left(U_{i} \otimes A_{i}\right)$ $=\left(\otimes_{i} U_{i}\right) \otimes A$. 
2.33 Definition. $G: U \otimes A \rightarrow V \otimes B$ will be called a map of twisted complexes (extending $g$ ) if it is of type $(0,0), g$-linear and

(i) $D G=G D$,

(ii) $G F_{p} \subset F_{p}$,

(iii) $L_{g}(G)=L_{g}(G-g)=G-g$.

The fact that the composition of two maps of twisted complexes is a map of twisted complexes will follow from 2.34 and 2.36 below.

2.34. Retaining the notations of 2.32, let $h: B \rightarrow C$ be a map of twisted algebras, $(W, C, D)$ a twisted resolution of $C$ and $H: V \otimes B \rightarrow W \otimes C$ a map of bigraded $K$-modules.

Lemma. (i) $h L_{g}(G)=L_{h g}(h G)$, (ii) $L_{h}(H) g=L_{h g}(H g)$.

The proof is easy; once again, we recall the "abuse of notation" involved in the use of the symbols $h, g$, cf. 2.322 .

2.35. This section is preliminary to the proof of 2.36 .

Let $u_{i} \in U_{i}(i=1, \ldots, n)$; since the product for the $U_{i}$ 's is the untwisted one we can write $u_{1} u_{2} \cdots u_{n}$ for $u_{1} \otimes \cdots \otimes u_{n}$ and $u_{1} u_{2} \cdots u_{n} u_{n}^{\prime}$ for $\left(u_{1} \otimes \cdots \otimes u_{n}\right) u_{n}^{\prime}$, $u_{n}^{\prime} \in U_{n}$. Also, let $g_{i} u_{i}=v_{i} \in V_{i}$. Now,

$$
\begin{aligned}
L_{g}(G)\left(u_{1} \cdots u_{n}\right)= & \Pi_{2} G\left(u_{1}\right) v_{2} \cdots v_{n}+\cdots+\Pi_{n} G\left(u_{1} \cdots u_{n-1}\right) v_{n} \\
& +\sum_{r=n+1}^{\infty} \Pi_{r} G\left(u_{1} \cdots u_{n}\right)
\end{aligned}
$$

from which (and the fact that $L_{g}(G)$ is " $g$-linear") we easily deduce the following formulas:

$$
\begin{aligned}
\Pi_{0} L_{g}(G) & =\Pi_{1} L_{g}(G)=0, \\
\Pi_{i} L_{g}(G) \Pi_{j} & =0 \text { if } i<j, \\
\Pi_{n} L_{g}(G)\left(u_{1} \cdots u_{n} u_{n}^{\prime}\right) & =\left\{\sum_{r=2}^{n-1} \Pi_{r} L_{g}(G)\left(u_{1} \cdots u_{n}\right)\right\}\left(\Pi_{n} v_{n}^{\prime}\right)+\left\{\Pi_{n} L_{g}(G)\left(u_{1} \cdots u_{n}\right)\right\} v_{n}^{\prime}
\end{aligned}
$$

and, in particular

$$
\begin{aligned}
& \Pi_{n} L_{g}(G)\left(u_{1} \cdots u_{n}\right)=\left\{\sum_{r=2}^{n-1} \Pi_{r} L_{g}(G)\left(u_{1} \cdots u_{n-1}\right)\right\}\left(\Pi_{n} v_{n}\right)+\left\{\Pi_{n} L_{g}\left(u_{1} \cdots u_{n-1}\right)\right\} v_{n}, \\
& \Pi_{i} L_{g}(G)\left(u_{1} \cdots u_{n}\right)=\Pi_{i} G\left(u_{1} \cdots u_{n}\right) \text { if } i>n .
\end{aligned}
$$

2.36. We retain the notations of $2.32,2.34,2.35$.

Lemma. (i) $L_{g}\left(L_{g}(G)\right)=L_{g}(G)$,

(ii) If $G=L_{g}(G), H=L_{h}(H)$, then $H G=L_{h g}(H G)$.

Proof. In both cases, we use the formulas of 2.35, and an induction on $n$. (i) is then nearly obvious.

To prove (ii) it clearly suffices to prove equality on $u_{1} u_{2} \cdots u_{n}$ where $u_{n} \in I\left(U_{n}\right)$. 
Let $g u_{i}=v_{i}, h v_{i}=w_{i}$. Clearly $\Pi_{n} u_{n}=u_{n}, \Pi_{n} v_{n}=v_{n}, \Pi_{n} w_{n}=w_{n}$ where $w_{n}$ or $v_{n}$ may be zero. Now

$$
\begin{aligned}
\Pi_{i} L_{h g}(H G)\left(u_{1} \cdots u_{n}\right)=0 & \text { if } i<n, \\
=\sum_{r=2}^{n} \Pi_{r}(H G)\left(u_{1} \cdots u_{n-1}\right) w_{n} & \text { if } i=n, \\
=\Pi_{i}(H G)\left(u_{1} \cdots u_{n}\right) & \text { if } i>n,
\end{aligned}
$$

using 2.35 and the inductive hypothesis. Now, since $G=L_{g}(G), H=L_{h}(H)$ we have $\Pi_{0} G=\Pi_{1} G=0, \Pi_{i} G \Pi_{j}=0$ if $i<j$ and similarly for $H$. Hence,

$$
\begin{aligned}
\Pi_{i} H G\left(u_{1} \cdots u_{n}\right) & =\Pi_{i} H \sum_{r=2}^{\infty} \Pi_{r} G\left(u_{1} \cdots u_{n}\right) \\
& =\Pi_{i} H \sum_{r=2}^{\infty} \Pi_{r} G\left(u_{1} \cdots u_{n}\right) .
\end{aligned}
$$

Now, $\Pi_{r} G\left(u_{1} \cdots u_{n}\right)=\Pi_{r} L_{g}(G)\left(u_{1} \cdots u_{n}\right)=0$ if $r<n$, and hence $\Pi_{i} H G\left(u_{1} \cdots u_{n}\right)=0$ if $i<n$.

It therefore remains to prove that $\Pi_{n} H G\left(u_{1} \cdots u_{n}\right)=\Pi_{n} H \Pi_{n} G\left(u_{1} \cdots u_{n}\right)$ is equal to $\Pi_{n} L_{n g}(H G)\left(u_{1} \cdots u_{n}\right)$. Now, since $G=L_{g}(G)$ we have

$$
\Pi_{n} G\left(u_{1} \cdots u_{n}\right)=\left\{\sum_{r=2}^{n} \Pi_{r} G\left(u_{1} \cdots u_{n-1}\right)\right\} v_{n}
$$

and hence, since $H=L_{h}(H)$,

$$
\Pi_{n} H \Pi_{n} G\left(u_{1} \cdots u_{n}\right)=\left\{\sum_{s=2}^{n} \sum_{r=2}^{n} \Pi_{s} H \Pi_{r} G\left(u_{1} \cdots u_{n-1}\right)\right\} w_{n} .
$$

Now, since $\Pi_{s} H \Pi_{r}=0$ if $s<r$, we can add all the terms $\Pi_{r} G$ for $r>n$ without changing anything. We get

$$
\left\{\sum_{s=2}^{n} \Pi_{s} H G\left(u_{1} \cdots u_{n-1}\right)\right\} w_{n}
$$

exactly as required. Q.E.D.

\section{Perturbations.}

3.0. Let $(U, A, D)$ be a twisted complex over the twisted algebra $(A, \Phi)$. Due to the convergence assumption 1.1(1) and 2.01(iii) there is a lower bound for the filtration of all nonzero elements of $(U \otimes A)_{m, n}$. Suppose now we have a sequence of maps of bimodules $G_{i}: U \otimes A \rightarrow V \otimes B(i=1,2, \ldots)$, where $(V, B, D)$ is a second twisted complex, each $G_{i}$ of type $(k, l)$ satisfying the condition

$$
G_{i} F_{p}(U \otimes A) \subset F_{p-\delta(i)}(V \otimes B)
$$

for all $p$, where $\delta(i) \rightarrow \infty$ as $i \rightarrow \infty$. Then it follows that for $w \in(U \otimes A)_{m, n}$ there is an integer $j$ such that $G_{i} w=0$ when $i \geqq j$. 
Using this remark, we shall say that $G_{i} \rightarrow 0$ as $i \rightarrow \infty$.

If $G_{i}: U \otimes A \rightarrow V \otimes B$, similarly, satisfies the condition

$$
\left(G_{j}-G_{i}\right) F_{p}(U \otimes A) \subset F_{p-\delta(i)}(V \otimes B)
$$

when $j \geqq i, \delta(i) \rightarrow \infty$, then for each $w \in(U \otimes A)_{m, n}$ there is an integer $j$ such that $G_{i} w=G_{i}, w$ for all $i, i^{\prime} \geqq j$. In this case, there is a well-defined map

$$
G: U \otimes A \rightarrow V \otimes B
$$

which can be regarded as $\lim _{i \rightarrow \infty} G_{i}$.

We can now explain the basic idea of this paper: Beginning with certain maps "appropriate" to an untwisted resolution $(U, A, d)$ of $\left(A, \Phi_{0}\right)$ we obtain sequences $G_{i}$ such that $\lim _{i \rightarrow \infty} G_{i}$ exists and is "appropriate" to a twisted resolution $(U, A, D)$ of $(A, \Phi)$. To shorten statements, we shall use the notations introduced in 2.1, 2.2, 2.3 without further explanation.

\subsection{Our first example is very simple.}

Proposition. Let $(U, A, d)$ be a resolution of $\left(A, \Phi_{0}\right)$ and $(U, A, D)$ a twisted complex over $(A, \Phi)(c f .2 .31)$, then $(U, A, D)$ is a resolution.

Proof. We need only prove the existence of a chain-homotopy for $D$. We define, inductively,

$$
\begin{aligned}
S_{1} & =s, \\
\Delta_{n} & =\left[D, S_{n}\right]-1+\Pi_{0} \quad(n \geqq 1), \\
S_{n+1} & =S_{n}-s \Delta_{n} .
\end{aligned}
$$

Let us write $\sigma=D-d$, so that by $2.31 \sigma F_{p} \subset F_{p-2}$. We shall prove by induction that $\Delta_{n} F_{p} \subset F_{p-n}$. Since $[d, s]=1-\Pi_{0}$, we have $\Delta_{1}=[D, s]-1+\Pi_{0}=[\sigma, s]$ which verifies the case $n=1$.

We now calculate:

$$
\begin{aligned}
{\left[D, S_{n+1}\right] } & =\left[D, S_{n}\right]-\left[D, s \Delta_{n}\right] \\
& =\Delta_{n}+1-\Pi_{0}-\left[d, s \Delta_{n}\right]-\left[\sigma, s \Delta_{n}\right] \\
& =\Delta_{n}+1-\Pi_{0}-d s \Delta_{n}-s \Delta_{n} d-\left[\sigma, s \Delta_{n}\right] \\
& =\Delta_{n}+1-\Pi_{0}-d s \Delta_{n}-s d \Delta_{n}+s\left[d, \Delta_{n}\right]-\left[\sigma, s \Delta_{n}\right] \\
& =\Delta_{n}+1-\Pi_{0}-\left(1-\Pi_{0}\right) \Delta_{n}+s\left[d, \Delta_{n}\right]-\left[\sigma, s \Delta_{n}\right] \\
& =1-\Pi_{0}+s\left[d, \Delta_{n}\right]-\left[\sigma, s \Delta_{n}\right]
\end{aligned}
$$

for $\Pi_{0} \Delta_{n}=\Pi_{0} D S_{n}+\Pi_{0} S_{n} D-\Pi_{0}+\Pi_{0} \Pi_{0}=0$ since $\Pi_{0} D=\Pi_{0} S_{n}=0$. Hence

$$
\Delta_{n+1}=s\left[d, \Delta_{n}\right]-\left[\sigma, s \Delta_{n}\right]
$$

Now $\left[d, \Delta_{n}\right]=\left[D, \Delta_{n}\right]-\left[\sigma, \Delta_{n}\right]$, and $\left[D, \Delta_{n}\right]=0$ as is easily verified; hence

$$
\Delta_{n+1}=-s\left[\sigma, \Delta_{n}\right]-\left[\sigma, s \Delta_{n}\right]
$$

from which $\Delta_{n+1} F_{p} \subset F_{p-n-1}$ is immediate. 
Hence $\Delta_{n} \rightarrow 0$ and $S_{n} \rightarrow S$ as $n \rightarrow \infty$, with $[D, S]-1+\Pi_{0}=0$. Q.E.D.

Note that the condition $L(D-l(d))=d-l(d)$ was not used in the above. The reason for this condition will appear later.

3.11 ADDENDUM. $S F_{p} \subset F_{p+1}$.

3.2. Continuing with the notations of $2.1,2.2,2.35$ let $\partial_{i}=l\left(d_{i}\right), u \in U_{p, q}$, $u^{\prime} \in U_{r, s}, a \in A_{t}, a^{\prime} \in A$.

$$
\begin{aligned}
\partial_{i}\left(u a \circ u^{\prime} a\right) & =(-1)^{t s} \partial_{i}\left(u u^{\prime} a \circ a^{\prime}\right) \\
& =(-1)^{t s} \partial_{i}\left(u u^{\prime}\right) \circ a \circ a^{\prime} \\
& =(-1)^{t s}\left\{\left(d_{i} u\right) u^{\prime}+(-1)^{p} u\left(d_{i} u^{\prime}\right)\right\} \circ a \circ a^{\prime},
\end{aligned}
$$

$\partial_{i}(u a) \circ u^{\prime} a^{\prime}+(-1)^{p} u a \circ \partial_{i}\left(u^{\prime} a^{\prime}\right)=\left(d_{i} u\right) \circ a \circ u^{\prime} a^{\prime}+(-1)^{p} u a \circ\left(d_{i} u^{\prime}\right) \circ a^{\prime}$

$$
\begin{aligned}
= & (-1)^{t s}\left(d_{i} u\right) u^{\prime} \circ a \circ a^{\prime}+(-1)^{p+t s} u\left(d_{i} u^{\prime}\right) \circ a \circ a^{\prime} \\
& +(-1)^{p} u\left\{a \circ\left(d_{i} u^{\prime}\right)-(-1)^{t s}\left(d_{i} u^{\prime}\right) \circ a\right\} \circ a^{\prime} .
\end{aligned}
$$

Hence,

$$
\partial_{i}\left(u a \circ u^{\prime} a^{\prime}\right)=\partial_{i}(u a) \circ u^{\prime} a^{\prime}+(-1)^{p} u a \circ \partial_{i}\left(u^{\prime} a^{\prime}\right)-(-1)^{p} u\left[a, d_{i} u^{\prime}\right] \circ a^{\prime}
$$

where the commutator $\left[a, d_{i} u^{\prime}\right]$ is taken, of course, in the sense of the product $\Phi$. Note that this "error-term" is zero if $a=1$.

3.31. Referring now to 2.32, let $G: U \otimes A \rightarrow V \otimes B$ again denote a map of bigraded $K$-modules. We define

and

$$
\theta_{i, g}(G)=\Phi\left\{t_{i+1} G \iota_{i} \otimes g^{i+1} \otimes \iota_{B} g\right\}
$$

$$
\theta_{g}(G)=\sum_{i=1}^{\infty} \theta_{i, g}(G)
$$

Note that $\theta_{g}(G)=L_{g}(s G)$, for $\Pi_{i+1} s=t_{i+1}$. Calculating, now, as in 2.35, 2.36, it is easy to verify the following formulas:

$$
\begin{array}{rlrl}
{\left[\partial_{j}, \theta_{i, g}(G)\right]=} & \theta_{i, g}\left[\partial_{j}, G\right] & & \text { if } j<i+1, \\
= & \Phi\left\{\Pi_{i+1} G \iota_{i} \otimes g^{i+1} \otimes \iota_{B} g\right\} & \\
& +X_{i, i+1, g}(G)+\theta_{i, g}\left[\partial_{i+1}, G\right] & & \text { if } j=i+1, \\
= & \theta_{i, g}\left[\partial_{j}, G\right]+X_{i, j, g}(G) & & \text { if } j>i+1 .
\end{array}
$$

If the "error-term" of 3.2 did not exist, neither would the functions $X_{i, j . g}(G)$ : These are simply the accumulated "error-terms" and are easily seen to satisfy the following conditions:

$$
\begin{aligned}
X_{i, j, g}(G)\left(u_{1} \cdots u_{n}\right) & =0 & & \text { if } n<j, \\
& =X_{i, j, g}(G)\left(u_{1} \cdots u_{j}\right) v_{j+1} \cdots v_{n} & & \text { if } n \geqq j,
\end{aligned}
$$

where, as before, $v_{k}=g u_{k}=g_{k} u_{k}$. From this we easily deduce

$$
L_{g}\left(X_{i, j, g}(G)\right)=X_{i, j, g}(G) .
$$


Finally, we notice the following: If $G F_{p} \subset F_{p-k}$, then $X_{i, j, g}(G) F_{p} \subset F_{p-k-1}$; this is true because, due to 1.1(2), the commutator "error-term" in 3.2, will have filtration at least one less than either of the two products.

Summing now over both indices and writing

$$
X_{g}(G)=\sum_{i=1}^{\infty} \sum_{j=i+1}^{\infty} X_{i, j, g}(G)
$$

we obtain, since $\sum \partial_{i}=l(d)$ :

3.32 Lemma. Writing $D_{1}=l(d),\left[D_{1}, \theta_{g}(G)\right]=\theta_{g}\left[D_{1}, G\right]+L_{g}(G)+X_{g}(G)$ where $X_{g}(G)$ satisfies the following conditions:

(i) $L_{g} X_{g}(G)=X_{g}(G)$.

(ii) If $G F_{p} \subset F_{p-k}$ for all $p$, then $X_{g}(G) F_{p} \subset F_{p-k-1}$.

3.4. As yet, we have not proved that twisted resolutions exist at all. We can now prove this; the main application of the next theorem, however, is not merely to prove existence: It is a practical means of finding resolutions for twisted algebras; that is the main reason for this entire paper.

TheOREM. Let $(U, A, d)$ be a resolution of $\left(A, \Phi_{0}\right)$, as in 2.1. Let $D_{1}=l(d)$ (cf. 2.2) and, inductively,

$$
D_{n+1}=D_{n}-\theta\left(D_{n}^{2}\right) \quad(n \geqq 1)
$$

where $\theta=\theta_{g}$ for $g=$ the identity on $A$, cf. 3.31. Then $\lim _{n \rightarrow \infty} D_{n}=D$ exists, and $(U, A, D)$ is a twisted resolution, cf. 2.31 .

Proof. From 1.1(2) we obtain immediately that $\left(D_{1}-d\right) F_{p} \subset F_{p-2}$; also, from $d^{2}=0$ we easily deduce $D_{1}^{2} F_{p} \subset F_{p-3}$; finally a detailed verification shows $L\left(D_{1}^{2}\right)$ $=D_{1}^{2}$. This is proved, using the method of $2.35,2.36$ and an induction on $n$.

We now prove inductively (i) $D_{n}^{2}=L\left(D_{n}^{2}\right)$, (ii) $D_{n}^{2} F_{p} \subset F_{p-n-2}$.

For $n=1$, we have just indicated the proofs.

$$
D_{n+1}^{2}=D_{n}^{2}-\left[D_{n}, \theta\left(D_{1}^{2}\right)\right]+\left\{\theta\left(D_{n}^{2}\right)\right\}^{2} .
$$

Now, let us write $D_{n}^{\prime}=D_{n}-D_{1}=-\theta\left(D_{1}^{2}\right)-\cdots-\theta\left(D_{n-1}^{2}\right)$.

$$
\begin{aligned}
{\left[D_{n}, \theta\left(D_{n}^{2}\right)\right] } & =\left[D_{1}, \theta\left(D_{n}^{2}\right)\right]+\left[D_{n}^{\prime}, \theta\left(D_{n}^{2}\right)\right] \\
& =\theta\left[D_{1}, D_{n}^{2}\right]+L\left(D_{n}^{2}\right)+X\left(D_{n}^{2}\right)+\left[D_{n}^{\prime}, \theta\left(D_{n}^{2}\right)\right] .
\end{aligned}
$$

Hence, remembering that $L\left(D_{n}^{2}\right)=D_{n}^{2}$,

$$
D_{n+1}^{2}=\theta\left[D_{1}, D_{n}^{2}\right]-X\left(D_{n}^{2}\right)-\left[D_{n}^{\prime}, \theta\left(D_{n}^{2}\right)\right]+\left\{\theta\left(D_{n}^{2}\right)\right\}^{2} .
$$

Now, since $\theta(G)=L(s G)$, it follows immediately from 2.36 that $L\left(D_{n+1}^{2}\right)=D_{n+1}^{2}$. Also, since $D_{n}^{\prime}=-\theta\left(D_{1}^{2}\right)-\cdots-\theta\left(D_{n-1}^{2}\right)$, the inductive hypothesis gives $D_{n}^{\prime} F_{p}$ $\subset F_{p-2}$. Also $\left[D_{1}, D_{n}^{2}\right]=\left[D_{n}-D_{n}^{\prime}, D_{n}^{2}\right]=-\left[D_{n}^{\prime}, D_{n}^{2}\right]$. Hence, the terms $\theta\left[D_{1}, D_{n}^{2}\right]$ and $\left[D_{n}^{\prime}, \theta\left(D_{n}^{2}\right)\right]$ decrease filtration by at least $n+3$; so does $\left\{\theta\left(D_{n}^{2}\right)\right\}^{2}$, since $2(n+1)$ $\geqq n+3$; finally $X\left(D_{n}^{2}\right) F_{p} \subset F_{p-n-3}$ by $3.32($ ii). 
This completes the inductive proof of (i) and (ii). Hence $D_{n}^{2} \rightarrow 0$ as $n \rightarrow \infty$ in the sense of 3.0 and

$$
D=\lim _{n \rightarrow \infty} D_{n}=\lim _{n \rightarrow \infty}\left\{D_{1}-\theta\left(D_{1}^{2}\right)-\cdots-\theta\left(D_{n-1}^{2}\right)\right\}
$$

exists, with $D^{2}=0$.

From the definitions, $D_{n}$ are all $A$-linear; hence so is $D$; $(D-d) F_{p} \subset F_{p-2}$ is immediate since $D_{1}-d=l(d)-d$ has this property, and $\theta\left(D_{n}^{2}\right) F_{p} \subset F_{p-n-1}$. Since $-D_{n}^{\prime}=\theta\left(D_{1}^{2}\right)+\cdots+\theta\left(D_{n-1}^{2}\right), L D_{n}^{\prime}=D_{n}^{\prime}$, whence $L\left(D-D_{1}\right)=D-D_{1}$.

This completes the proof of the fact that $(U, A, D)$ is a twisted complex over $(A, \Phi)$. Hence it is a resolution, by 3.1. Q.E.D.

3.5. Next, by a similar method, we prove the existence of maps of resolutions. Let $(U, A, D),(V, B, D)$ be twisted complexes, $g: A \rightarrow B$ a map of twisted algebras, cf. 1.1. As in 2.32, we extend $g$ to a map $g: U \otimes A \rightarrow V \otimes B$. Clearly $g$ is a map of bigraded modules of type $(0,0)$ such that $d g=g d$, i.e. $[g, d]=0$. Writing, again, $D_{1}=l(d)$, we easily see that, also, $\left[g, D_{1}\right]=0$; note $g=l_{g}(g)$. Finally, assume that $(V, B, D)$ is a resolution.

THEOREM. With these data, define inductively

$$
G_{1}=g, \quad G_{n+1}=G_{n}-\theta_{g}\left[D, G_{n}\right] \quad(n \geqq 1)
$$

then $\lim _{n \rightarrow \infty} G_{n}=G$ exists; and $G$ is a map of complexes extending $g, c f .2 .33$.

Proof. We prove inductively that (i) $\left[D, G_{n}\right]=L_{g}\left[D, G_{n}\right]$ and (ii) $\left[D, G_{n}\right] F_{p}$ $\subset F_{p-n-1}$.

Writing, again, $D^{\prime}=D-D_{1}$, we have $D^{\prime}=L D^{\prime}$. Hence, by $2.34, D^{\prime} g=\left(L D^{\prime}\right) g$ $=L_{g}\left(D^{\prime} g\right)$ and, similarly, $g D^{\prime}=L_{g}\left(g D^{\prime}\right)$, whence $\left[D^{\prime}, g\right]=L_{g}\left[D^{\prime}, g\right]$. Also, $\left[D_{1}, g\right]$ $=0$. Hence $[D, g]=L_{g}[D, g]$.

Since $D^{\prime} F_{p} \subset F_{p-2}$ and $g F_{p} \subset F_{p},[D, g] F_{p}=\left[D^{\prime}, g\right] F_{p} \subset F_{p-2}$; and the verification for $n=1$ is complete. Now

$$
\begin{aligned}
{\left[D, G_{n+1}\right] } & =\left[D, G_{n}\right]-\left[D_{1}, \theta_{g}\left[D, G_{n}\right]\right]-\left[D^{\prime}, \theta_{g}\left[D, G_{n}\right]\right] \\
& =\left[D, G_{n}\right]-\theta_{g}\left[D_{1},\left[D, G_{n}\right]\right]-L_{g}\left[D, G_{n}\right]-X_{g}\left[D, G_{n}\right]-\left[D^{\prime}, \theta_{g}\left[D, G_{n}\right]\right] \\
& =\theta_{g}\left[D,\left[D, G_{n}\right]\right]-X_{g}\left[D, G_{n}\right]-\left[D^{\prime}, \theta_{g}\left[D, G_{n}\right]\right]
\end{aligned}
$$

from the inductive hypothesis, and using $D_{1}=D-D^{\prime}$.

(i) now follows easily from 2.34 and 2.36; and (ii) from the inductive hypothesis and $D^{\prime} F_{p} \subset F_{p-2}$. The rest of the proof is now exactly like the last part of 3.4.

3.6. It is interesting to observe that the results of $3.4,3.5$ can be written as infinite series. Writing $x_{1}=D_{1}, x_{i}=-\theta\left(D_{i-1}^{2}\right)(i \geqq 2), y_{1}=g, y_{i}=-\theta_{g}\left[D, G_{i-1}\right]$ we have $D=\sum_{i=1}^{\infty} x_{i}, G=\sum_{i=1}^{\infty} y_{i}$, where $x_{i} F_{p} \subset F_{p-i}, y_{i} F_{p} \subset F_{p-i+1}$.

4. Spectral sequences. The filtration $F$ leads to various spectral sequences. Since we want to introduce at least one other filtration, we consider filtrations in slightly greater generality. 
4.11 Definition. If $(A, \varphi)$ is an algebra we call $(A, \varphi, \mathfrak{F})$ a filtered algebra or $\mathfrak{F}$ a filtration of $(A, \varphi)$, if $\mathfrak{F}$ is an increasing filtration of the module $A$ such that

$$
\varphi\left(\mathfrak{F}_{p} A \otimes \mathfrak{F}_{q} A\right) \subset \mathfrak{F}_{p+q}(A) .
$$

4.12 Definition. Let $(A, \varphi)$ be an algebra, $\mathfrak{F}$ a filtration of $(A, \varphi)$. Let $(U, A, d, s)$ be a resolution of $(A, \varphi)$ (cf. 2.0). A filtration $\left\{\mathfrak{F}_{p}(U \otimes A)\right\}=\mathfrak{F}$ of the module $U \otimes A$ will be called an admissible extension of $\mathfrak{F} \mid A$ if

(i) $\mathfrak{F}_{p}(U \otimes A) \cap A=\mathfrak{F}_{p} A$.

(ii) $d \mathfrak{F}_{p} \subset \mathfrak{F}_{p-1}, s \mathfrak{F}_{p} \subset \mathfrak{F}_{p+1}$.

(iii) Given integers $(m, n)$ there is an integer $p(m, n)$ such that $\mathfrak{F}_{p} \cap(U \otimes A)_{m, n}$ $=0$ when $p<p(m, n)$.

4.13. Note that if $(A, \varphi, \mathfrak{F})$ is a filtered algebra the associated graded module is an algebra $E^{0} A ; E^{0} A$ is bigraded with

$$
\left(E^{0} A\right)_{p, s}=\frac{\mathfrak{F}_{p} \cap A_{p+s}}{\mathfrak{F}_{p-1} \cap A_{p+s}}
$$

As a graded algebra, we take $p+s$ as the grading of $E^{0} A$.

4.2. If $A$ is a graded algebra, we write, as usual, $H^{k, t}(A)=\operatorname{Ext}_{A}^{k, t}(K, K)$, where $k$ is the homological and $t$ the "internal" dimension; cf. [4, p. 298].

TheOREM. Let $(A, \varphi, \mathfrak{F})$ be a filtered algebra. Let $(U, A, d, s)$ be a resolution of $(A, \varphi)$, and let $\mathfrak{F}$ also denote an admissible extension of $\mathfrak{F}$. Then $\mathfrak{F}$ induces a convergent spectral sequence $\left(E_{r}^{p, q, t}, d_{r}\right)$ with $d_{r}: E_{r}^{p, q, t} \rightarrow E_{r}^{p+r, q-r+1, t}$ and such that

(i) $E_{2}^{p, q, t}=H^{p+q-t, t}\left(E^{0} A\right)$,

(ii) $E_{\infty}^{p, q, t}=E^{0}\left(H^{p+q-t, t}(A)\right)$ for some filtration of $H^{p+q-t, t}(A)$.

Proof. Since $d \mathfrak{F}_{p} \subset \mathfrak{F}_{p-1}$, in the spectral sequence $\left(E^{r}, d^{r}\right)$ induced by $\mathfrak{F}, d^{0}=0$ and $E^{0}=E^{1}$. Since $s \mathfrak{F}_{p} \subset \mathfrak{F}_{p+1}, s$ induces a chain-homotopy in $E^{1}$ which therefore is a resolution of $E^{0} A$. Applying now the functor $\operatorname{Hom}_{A}(, K)$, the statement (i) is apparent.

4.21 ADDENDUM. In the same situation, if $\left(U^{\prime} A, d^{\prime}, s^{\prime}\right)$ denotes a second resolution $(A, \varphi)$, and $\mathfrak{F}^{\prime}$ an admissible extension of $\mathfrak{F}$ for $U^{\prime} \otimes A$, then the spectral sequences of 4.2 induced by $\mathfrak{F}$ and $\mathfrak{F}^{\prime}$ are isomorphic from $E^{2}$ on.

Proof. Due to condition (ii) in 4.12, the chain-map $U \otimes A \rightarrow U^{\prime} \otimes A$ constructed in the standard comparison theorem is filtration-preserving. This map therefore induces isomorphisms throughout the spectral sequence from $E^{2}$ on, since the necessary chain-homotopies occur in $E^{1}$.

4.3 ExAMPLES. (i) The filtration $F$ of 2.1 , associated with a twisted resolution of a twisted algebra, of 2.31 , is admissible. For reference we shall call this filtration the weighted dimension filtration.

Note that in this case $E^{0} A \approx\left(A, \Phi_{0}\right)$ by $1.1(2)$.

(ii) Let $(A, \varphi)$ be an algebra. The augmentation filtration $\mathfrak{F}$ is defined by letting $\mathfrak{F}_{-p}(A)$ be $\varphi(I A \otimes \cdots \otimes I A)$ ( $p$ factors) for $p<0$ and $\mathfrak{F}_{p}(A)=A$ for $p \geqq 0$. If $A$ 
is projective, this can be extended to the bar-construction, by letting $\mathfrak{F}_{p}$ be the submodule generated by all elements $a\left[a_{1}, a_{2}, \ldots, a_{n}\right]$ for which $a \in \mathfrak{F}_{q}(A)$, $a_{i} \in \mathfrak{F}_{p_{i}}(A)$ and $q+p_{1}+\cdots+p_{n}+n \leqq p$.

This filtration is admissible; it has been introduced and much considered by May; we call it the May-filtration; the corresponding spectral sequence 4.2 we call the May-spectral sequence.

(iii) In exactly the same way, the filtration $F$ of 1.0 can also be introduced into the bar-construction of $(A, \Phi)$. This case, exactly as (i) above, has $E^{0} A=\left(A, \Phi_{0}\right)$.

4.4. For computations, the May-spectral sequence is convenient; since it is not that of a twisted resolution it is, in general, not related to the methods of $\S 3$; in particular, if we find $D$ by the method of 3.4 , there is no reason to suppose that it will be "compatible" with the May-filtration of the bar-construction.

In certain cases, however, something of this sort is true. Suppose $\left(\left\{A_{i}, \varphi_{i}\right\}, \Phi\right)$ is a twisted algebra, cf. 1.1. Let $\mathfrak{F}^{i}$ be a filtration of $A_{i}$. We define the associated weighted filtration $\mathfrak{F}$ of $A=\bigotimes_{i} A_{i}$ as follows: $\mathfrak{F}_{p} A$ is generated by all monomials $a_{1} \otimes a_{2} \otimes \cdots \otimes a_{n}$ where $a_{i} \in \mathfrak{F}_{p_{i}}^{i}\left(A_{i}\right)$ and $p_{1}+2 p_{2}+\cdots+n p_{n} \leqq p$. Thus, we obtain the filtration of 1.0 if each $A_{i}$ is filtered by negative dimension. If, cf. (2.1), $\left(U_{i}, A_{i}, d_{i}\right)$ is a resolution of $\left(A_{i}, \varphi_{i}\right)$, suppose that $\mathfrak{F}^{i}$ has been extended to $U_{i} \otimes A_{i}$ so as to satisfy condition 4.12 . We can then extend $\mathfrak{F}$ by using the same method, to the associated weighted filtration of $U \otimes A$; this will satisfy 4.12 in relation to the untwisted differential and homotopy $d$ and $s$.

Suppose now that the weighted filtration satisfies $\Phi\left(\mathfrak{F}_{p} \otimes \mathfrak{F}_{q}\right) \subset \mathfrak{F}_{p+q}$; in general, this will not be the case; though certainly $\Phi_{0}\left(\mathfrak{F}_{p} \otimes \mathfrak{F}_{q}\right) \subset \mathfrak{F}_{p+q}$.

Then it is easily verified that if $G: U \otimes A \rightarrow U \otimes A$ is such that $G \mathfrak{F}_{p} \subset \mathfrak{F}_{p+k}$ (for some fixed $k$ ), then

$$
\begin{array}{ll}
\left(L_{g} G\right) \mathfrak{F}_{p} \subset \mathfrak{F}_{p+k} & \text { (cf. 2.32), } \\
\left(\theta_{g} G\right) \mathfrak{F}_{p} \subset \mathfrak{F}_{p+k+1} & \text { (cf. 3.31). }
\end{array}
$$

Hence, it is easily seen that the behavior with respect to filtration is not changed by the sequence of perturbations in 3.4 or 3.5 .

We have proved:

4.41 LemMA. The associated weighted filtration $\mathfrak{F}$ obtained from a sequence of admissible filtrations is admissible (in relation to $S, D$, of $3.1,3.4$ ), provided

$$
\Phi\left(\mathfrak{F}_{p} \otimes \mathfrak{F}_{q}\right) \subset \mathfrak{F}_{p+q} .
$$

4.42 Remark. Note that for the case of the weighted dimension filtration the condition is a consequence of the stronger condition 1.1(2). This condition will in general not be satisfied by the admissible filtrations of 4.41 . Hence, even though one may prefer, in calculations, to use another filtration, the weighted dimension filtration is essential for the convergence proofs of $\S 3$.

4.5 Application. The Steenrod algebra $(\bmod p)$ is a twisted algebra; $A_{i}$ is the 
divided polynomial algebra generated by the element dual to $\xi_{i}$ tensored by the exterior algebra generated by the element dual to $\tau_{i}$, using the notation of Milnor, [10]; in the case $p=2, \tau_{i}$ is absent. If $R=\left(r_{1}, \ldots, r_{k}\right)$ is a sequence of integers, the element dual to $\xi_{1}^{r_{1}} \cdots \xi_{k}^{r_{k}}$ is denoted by $P(R)$; the element dual to $\tau_{i}$ is denoted by $Q_{i}$.

4.51 Lemma. With this representation of the Steenrod algebra, the augmentation filtration and the weighted filtration associated with the augmentation filtrations of the $A_{i}$ are identical.

Proof. If $x \in A$, following May, we write $w(x)=n$ if

$$
x \in \Phi\left((I A)^{n}\right) \text { and } x \notin \Phi\left((I A)^{n+1}\right) .
$$

If $R=\left(r_{1}, \ldots, r_{k}\right)$ and $r_{i}=\sum_{j=0}^{\infty} a_{i j} p^{i}$ is the $p$-adic expansion of $r_{i}$, then we have Theorem I. 2.2 of [7]:

$$
w(P(R))=\sum_{i=1}^{\infty} \sum_{j=0}^{\infty} i a_{i j}
$$

the " $w$ " of the dual of $\xi_{i}^{r_{i}}$ in $A_{i}$ is easily seen to be exactly $\sum_{j=0}^{\infty} a_{i j}$. (Recall that $A_{i}$ is a divided polynomial algebra.) Hence $w(P(R))$ is obtained from these numbers exactly by our weighting. Formula (iv) in Theorem II. 1.1, namely $Q_{i+1}=\left[P^{p^{i}}, Q_{i}\right]$, gives, by an evident induction, $w\left(Q_{i}\right)=i$, while the weight of $Q_{i}$ in $A_{i}$ is clearly 1 . Q.E.D.

4.52 COROLlary. The weighted augmentation filtration satisfies the condition of 4.41 in the case of the Steenrod algebra.

4.53 COROLLARY. The spectral sequence of 4.2 obtained by extending the (weighted) augmentation filtration of the Steenrod algebra to any admissible filtration of a twisted resolution is isomorphic, from $E^{2}$ on, to the May spectral sequence.

Proof. 4.21.

4.6. If $A$ is a Hopf algebra, it is well known that the diagonal $\psi: A \rightarrow A \otimes A$ can be extended to a map of resolutions $\psi: U \otimes A \rightarrow U \otimes U \otimes A \otimes A \approx U$ $\otimes A \otimes U \otimes A$; and that this map induces the multiplicative structure of Ext. If $\psi$ is filtration-preserving, i.e., if $\psi \mathfrak{F}_{p} \subset \sum_{i+j=p} \mathfrak{F}_{i} \otimes \mathfrak{F}_{j}$, if $U \otimes A$ has an admissible extension of the filtration $\mathfrak{F}$ of $A$, cf. 4.12 , then, by the same argument as in the proof of $4.21, \bar{\psi}$ is filtration-preserving. This implies that the spectral sequence, cf. 4.2 , is multiplicative:

4.61 Addendum to Theorem 4.2. Under the conditions of 4.2, if $(A, \varphi, \psi)$ is a Hopf algebra and if $\psi$ is filtration-preserving, we have the further results:

(iii) $\left(E_{r}, d_{r}\right)$ has the structure of a trigraded differential algebra.

(iv) The isomorphisms of 4.2(i), (ii) are isomorphisms of algebras

$$
E^{2} \rightarrow H^{*}\left(E^{0} A\right), \quad E_{\infty} \rightarrow E^{0}\left(H^{*} A\right) .
$$


5. The subalgebras $\mathscr{A}_{(t)}$ of the Steenrod algebra $\mathscr{A}$. In this and the following sections we use the techniques developed earlier to give a partial computation of $\operatorname{Ext}_{\mathscr{A}_{(3)}}\left(Z_{2}, Z_{2}\right)$ where $\mathscr{A}_{(t)}$ is the subalgebra of the (mod 2) Steenrod algebra $\mathscr{A}$ generated by $S q^{1}, S q^{2}, \ldots, S q^{2 t}$, cf. [9, p. 23].

5.1. We start by giving the

5.11 Definition. $q_{i, j}$ is the dual of $\xi_{i}^{j}$ in Milnor's description of $\mathscr{A}$ [10].

NOTE. In particular $q_{1, j}=S q^{j}$.

5.12 ThEOREM (MILNOR). Every element in $\mathscr{A}$ can be written uniquely as a sum of elements of the form $q_{i_{1} j_{1}} \cdots q_{i_{r} j_{r}}$ with $0<i_{1} \leqq i_{2} \leqq \cdots \leqq i_{r}, j_{k}<j_{k+1}$ if $i_{k}=i_{k+1}$ and each $j_{k}$ is a power of 2 .

Thus we obtain a complete knowledge of the multiplicative structure of $\mathscr{A}$ if we can express all squares $\left(q_{i, 2^{j}}\right)^{2}$ and all commutators $\left[q_{i, 2^{j}}, q_{k, 2^{l}}\right]$ in the standard form 5.12. The subalgebra $\mathscr{A}_{(t)}$ is generated by all $q_{i, 2}$ where $j \leqq t, i+j \leqq t+1$. $Q_{n}$, in the notation of 1.22 , is generated by $q_{i, j}$ with $i<n$. The twisting function of $Q_{n+1}=Q_{n} \otimes A_{n+1}$ is obtained by writing all commutators of generators in $Q_{n}$ and observing the terms with $q_{n+1, j}$.

5.2. The algebra $\mathscr{A}_{(3)}$. As a twisted tensor-product, $\mathscr{A}_{(3)}$ has 10 generators, namely $q_{1,1}, q_{1,2}, q_{1,4}, q_{1,8}, q_{2,1}, q_{2,2}, q_{2,4}, q_{3,1}, q_{3,2}, q_{4,1}$. To give the complete structure we need 10 squares and 45 commutators. However, these are all consequences of the following basic relations:

$$
\begin{gathered}
{\left[S q^{1}, S q^{2}\right]=q_{2,1}, \quad\left[S q^{2}, S q^{4}\right]=q_{2,2}, \quad\left[S q^{4}, S q^{8}\right]=q_{2,4}+S q^{3} q_{2,1} q_{2,2},} \\
{\left[S q^{1}, q_{2,2}\right]=q_{3,1}, \quad\left[S q^{2}, q_{2,4}\right]=S q^{1} q_{2,2} q_{3,1}+q_{3,2}} \\
{\left[S q^{1}, q_{3,2}\right]=q_{4,1}, \quad\left[S q^{1}, S q^{4}\right]=S q^{1} q_{2,1}} \\
{\left[S q^{1}, S q^{8}\right]=S q^{2} S q^{4} q_{2,1}+S q^{2} q_{3,1}, \quad\left[S q^{2}, S q^{8}\right]=q_{2,1} q_{3,1}+S q^{4} q_{2,2}} \\
\left(S q^{1}\right)^{2}=0, \quad\left(S q^{2}\right)^{2}=S q^{1} q_{2,1}, \quad\left(S q^{4}\right)^{2}=S q^{2} q_{2,2} \\
\left(S q^{8}\right)^{2}=S q^{4} q_{2,4}+S q^{7} q_{2,1} q_{2,2}+\left(S q^{3}+q_{2,1}\right) q_{2,2} q_{3,1}
\end{gathered}
$$

6. The perturbations for $\mathscr{A}_{(3)}$. Over $Z_{2}$ a formalism for handling the systems of perturbations of $D_{1}$ is fairly easy to arrange. In particular, for $\mathscr{A}_{(3)}$ the complex we start with is a divided polynomial algebra

6.01. $\Lambda\left(k_{4,1}, k_{3,2}, k_{3,1}, k_{2,4}, k_{2,2}, k_{2,1}, k_{1,8}, k_{1,4}, k_{1,2}, k_{1,1}\right)$ and any element in it is a sum of monomials of the form

$$
\left(k_{4,1}\right)_{j_{1}}\left(k_{3,2}\right)_{j_{2}} \cdots\left(k_{1,1}\right)_{j_{10}} .
$$

6.1 Definition. The ordered 10-tuple of integers (positive, negative or zero) $\left(i_{1}, \ldots, i_{10}\right)$ denotes the operator which sends $\left(k_{4,1}\right)_{j_{1}} \cdots\left(k_{1,1}\right)_{j_{10}}$ into the monomial $\left(k_{4,1}\right)_{j_{1}+i_{1}} \cdots\left(k_{1,1}\right)_{j_{10}+i_{10}}$; where $\left(k_{*, *}\right)_{i}=1$ if $i=0$ and $=0$ if $i<0$.

Such operators are provided with coefficients from $\mathscr{A}_{(3)}$ in the evident way, and iterating two operators with coefficients $\alpha\left(i_{1} \cdots i_{10}\right), \beta\left(j_{1} \cdots j_{10}\right)$ we obtain $\alpha \beta\left(i_{1}+j_{1}, \ldots, i_{10}+j_{10}\right)$. These operators would seem to be sufficient to describe the 
perturbing differentials. However, this is not quite true since the linearization operator of 2.2 involves the multiplication in the divided polynomial algebra as well as that in $\mathscr{A}_{(3)}$. Hence, in iterating two operators, binomial coefficients are introduced which depend on the monomial on which the operator is operating as well as upon the operator.

For example, if we start with the element $\left(k_{2,1}\right)_{i}\left(k_{1,2}\right)_{j}\left(k_{1,1}\right)_{s}$ (appropriate to the subalgebra of $\mathscr{A}_{(3)}$ generated by $\left.S q^{1}, S q^{2}\right)$, we have the formula

$$
\begin{aligned}
D_{1}\left(\alpha\left(k_{2,1}\right)_{i}\left(k_{1,2}\right)_{j}\left(k_{1,1}\right)_{s}\right)=\alpha\{ & q_{2,1}\left(k_{2,1}\right)_{i-1}\left(k_{1,2}\right)_{j}\left(k_{1,1}\right)_{s} \\
& \left.+S q^{2}\left(k_{2,1}\right)_{i}\left(k_{1,2}\right)_{j-1}\left(k_{1,1}\right)_{s}+S q^{1}\left(k_{2,1}\right)_{i}\left(k_{1,2}\right)_{j}\left(k_{1,1}\right)_{s-1}\right\}
\end{aligned}
$$

which corresponds to the operation of

$$
q_{2,1}(-1,0,0)+S q^{2}(0,-1,0)+S q^{1}(0,0,-1)
$$

on the element. Starting the perturbation process, we find

$$
\begin{aligned}
\theta\left(D_{1}^{2}\right)\left(\alpha\left(k_{2,1}\right)_{i}\left(k_{1,2}\right)_{j}\left(k_{1,1}\right)_{s}\right)=\alpha\left\{\left(\begin{array}{c}
i+1 \\
1
\end{array}\right)\left(k_{2,1}\right)_{i+1}\left(k_{1,2}\right)_{j-1}\left(k_{1,1}\right)_{s-1}\right. \\
\left.+\left(\begin{array}{c}
i+1 \\
1
\end{array}\right) S q^{1}\left(k_{2,1}\right)_{i+1}\left(k_{1,2}\right)_{j-2}\left(k_{1,1}\right)_{s}\right\}
\end{aligned}
$$

and we write

$$
\theta\left(D_{1}^{2}\right)=\left(\begin{array}{c}
*+1 \\
1
\end{array}\right)\left[(1,-1,-1)+S q^{1}(1,-2,0)\right] .
$$

This is a typical example of how a binomial coefficient is introduced. Continuing the process we find

$$
\theta\left(D_{2}^{2}\right)\left(\left(k_{2,1}\right)_{i}\left(k_{1,2}\right)_{j}\left(k_{1,1}\right)_{s}\right)=\alpha\left(\begin{array}{c}
i+2 \\
2
\end{array}\right)\left(k_{2,1}\right)_{i+2}\left(k_{1,2}\right)_{j-3}\left(k_{1,1}\right)_{s}
$$

and we write

$$
\theta\left(D_{2}^{2}\right)=\left(\begin{array}{c}
*+2 \\
2
\end{array}\right)(2,-3,0)
$$

Here the process stops, and $D=D_{1}+\theta\left(D_{1}^{2}\right)+\theta\left(D_{2}^{2}\right)$ gives the differential for the algebra $\mathscr{A}_{(1)}$. From this, the calculation of $\operatorname{Ext}_{\mathscr{A}_{(1)}}\left(Z_{2}, Z_{2}\right)$ becomes routine.

More generally, we have the evident

6.11 Proposition. The operators in the perturbed differential are sums of operators $\alpha \rho\left(i_{1} \cdots i_{10}\right)$ where

$\alpha \rho\left(i_{1} \cdots i_{10}\right)\left[\beta\left(k_{4,1}\right)_{j_{1}} \cdots\left(k_{1,1}\right)_{j_{10}}\right]=\beta \bar{\rho}\left(i_{1} \cdots i_{10}, j_{1} \cdots j_{10}\right) \alpha\left[\left(k_{4,1}\right)_{j_{1}+i_{1}} \cdots\left(k_{1,1}\right)_{j_{10}+i_{10}}\right]$ where $\bar{\rho}\left(i_{1} \cdots i_{10}, j_{1} \cdots j_{10}\right)$ is a product of binomial coefficients and belongs to $Z_{2}$.

6.2. Before proceeding further we express the operator $\theta$ of 3.21 in this notation:

$$
\theta\left[\alpha \rho\left(i_{1}, \ldots, i_{10}\right)\right]=\alpha^{\prime} \rho\left(i_{1}, \ldots, i_{k}+1, \ldots, i_{10}\right)
$$

provided $\alpha=\alpha^{\prime} q_{(k)}$ written in the ordering of 5.12 and $q_{(k)}$ is the $q_{i, j}$ in the $k$ th position. 
6.3. We now proceed directly to the calculations. To start

$$
\begin{aligned}
D_{1}= & q_{4,1}(-1,0,0, \ldots, 0)+q_{3,2}(0,-1,0, \ldots, 0) \\
& +q_{3,1}(0,0,-1,0 \cdots)+q_{2,4}(0,0,0,-1,0, \ldots, 0) \\
& +q_{2,2}(0,0,0,0,-1,0 \cdots) \\
& +q_{2,1}(0,0,0,0,0,-1,0 \cdots)+S q^{8}(0,0,0,0,0,0,-1,0,0,0) \\
& +S q^{4}(0,0,0,0,0,0,0,-1,0,0)+S q^{2}(0,0,0,0,0,0,0,0,-1,0) \\
& +S q^{1}(0,0,0,0,0,0,0,0,0,-1) .
\end{aligned}
$$

The first perturbation comes from evaluating $D_{1}^{2}$ and we find

$$
\begin{aligned}
\overline{\boldsymbol{x}}_{2}= & \bar{\rho}(6)(0,0,0,0,0,1,0,0,-1,-1)+\bar{\rho}(5)(0,0,0,0,1,0,0,-1,-1,0) \\
& +\bar{\rho}(4)(0,0,0,1,0,0,-1,-1,0,0)+\bar{\rho}(3)(0,0,1,0,-1,0,0,0,0,-1) \\
& +\bar{\rho}(3)(0,0,1,0,0,-1,0,-1,0,0)+\bar{\rho}(2)(0,1,0,-1,0,0,0,0,-1,0) \\
& +\bar{\rho}(1)(1,-1,0,0,0,0,0,0,0,-1)+\bar{\rho}(1)(1,0,0,-1,0,-1,0,0,0,0) \\
& +\bar{\rho}(1)(1,0,-1,0,0,0,-1,0,0,0)+\bar{\rho}(2)(0,1,0,0,-1,0,-1,0,0,0)
\end{aligned}
$$

where

$$
\bar{\rho}\left(j_{r}\right)\left(i_{1} \cdots i_{10}\right)\left(\left(k_{4,1}\right)_{j_{1}} \cdots\left(k_{4,1}\right)_{j_{10}}\right)=\left(\begin{array}{c}
i_{r}+j_{r} \\
i_{r}
\end{array}\right)\left(k_{4,1}\right)_{j_{1}+i_{1}} \cdots\left(k_{1,1}\right)_{j_{10}+i_{10}},
$$

and of course $\overline{\boldsymbol{x}}$ is the differential obtained after tensoring over $\mathscr{A}_{(3)}$ with $Z_{2}$. Similarly the terms in $x_{3}$ come about by considering $x_{1}^{2}+\left[x_{1}, x_{2}\right]+x_{2}^{2}$ and taking $\theta$ on this (cf. 3.6).

6.33. $\bar{x}_{3}$ is given by:

\begin{tabular}{l|l|l|l|l|l|l|l|l|l|l}
\hline Coefficient & $k_{4,1}$ & $k_{3,2}$ & $k_{3,1}$ & $k_{2,4}$ & $k_{2,2}$ & $k_{2,1}$ & $k_{1,8}$ & $k_{1,4}$ & $k_{1,2}$ & $k_{1,1}$ \\
\hline $\bar{\rho}(5) \cdot \bar{\rho}(6)$ & & & & & 1 & 1 & & -2 & & -1 \\
\hline $\bar{\rho}(6) \cdot \bar{\rho}(6)$ & & & & & & 2 & & -1 & & -2 \\
\hline $\bar{\rho}(3) \cdot \bar{\rho}(6)$ & & & 1 & & & 1 & -1 & & & -2 \\
\hline $\bar{\rho}(3) \cdot \bar{\rho}(3)$ & & & 2 & -1 & & & & & & -2 \\
\hline $\bar{\rho}(3) \cdot \bar{\rho}(3)$ & & & 2 & & -2 & & & & -1 & \\
\hline $\bar{\rho}(1) \cdot \bar{\rho}(3)$ & 1 & -1 & 1 & & -1 & & & & -1 & \\
\hline $\bar{\rho}(1) \cdot \bar{\rho}(1)$ & 2 & -2 & & & & & & & -1 & \\
\hline $\bar{\rho}(6) \cdot \bar{\rho}(6)$ & & & & & & 2 & & & -3 & \\
\hline $\bar{\rho}(3) \cdot \bar{\rho}(6)$ & & & 1 & & -1 & 1 & & & -2 & \\
\hline $\bar{\rho}(1) \cdot \bar{\rho}(3)$ & 1 & -1 & 1 & & & & & & -2 & \\
\hline
\end{tabular}




\begin{tabular}{c|c|c|c|c|c|c|c|c|c|c}
\hline Coefficient & $k_{4,1}$ & $k_{3,2}$ & $k_{3,1}$ & $k_{2,4}$ & $k_{2,2}$ & $k_{2,1}$ & $k_{1,8}$ & $k_{1,4}$ & $k_{1,2}$ & $k_{1,1}$ \\
\hline $\bar{\rho}(3) \cdot \bar{\rho}(3)$ & & & 2 & & -1 & & & -2 & & \\
\hline $\bar{\rho}(1) \cdot \bar{\rho}(3)$ & 1 & & 1 & -1 & -1 & & & -1 & & \\
\hline $\bar{\rho}(2) \cdot \bar{\rho}(2)$ & & 2 & & -2 & & & & -1 & & \\
\hline $\bar{\rho}(5) \cdot \bar{\rho}(5)$ & & & & & 2 & & & -3 & & \\
\hline $\bar{\rho}(2) \cdot \bar{\rho}(5)$ & & 1 & & -1 & 1 & & & -2 & & \\
\hline $\bar{\rho}(1) \cdot \bar{\rho}(3)$ & 1 & & 1 & & -1 & & -2 & & & \\
\hline $\bar{\rho}(2) \cdot \bar{\rho}(2)$ & & 2 & & -1 & & & -2 & & & \\
\hline $\bar{\rho}(1) \cdot \bar{\rho}(1)$ & 2 & & -1 & & & & -2 & & & \\
\hline $\bar{\rho}(3) \cdot \bar{\rho}(3)$ & & & 2 & & & & -1 & -1 & -1 & \\
\hline $\bar{\rho}(1) \cdot \bar{\rho}(3)$ & 1 & & 1 & -1 & & & -1 & & & \\
\hline $\bar{\rho}(4) \cdot \bar{\rho}(5)$ & & & & 1 & 1 & & -2 & & -1 & \\
\hline $\bar{\rho}(4) \cdot \bar{\rho}(4)$ & & & & 2 & & & -1 & & -2 & \\
\hline $\bar{\rho}(3) \cdot \bar{\rho}(5)$ & & & 1 & & 1 & -1 & -1 & & -1 & \\
\hline $\bar{\rho}(4) \cdot \bar{\rho}(4)$ & & & & 2 & & & -3 & & & \\
\hline $\bar{\rho}(3) \cdot \bar{\rho}(3)$ & & & 1 & 1 & -1 & & -2 & & & \\
\hline $\bar{\rho}(1) \cdot \bar{\rho}(1)$ & 2 & & & -2 & -1 & & & & & \\
\hline $\bar{\rho}(3) \cdot \bar{\rho}(3)$ & & & 2 & & & -2 & -1 & & & \\
\hline & & & & & & & & \\
\hline
\end{tabular}

7. The $E_{2}, E_{3}$ terms of the May spectral sequence.

7.1. The $E_{1}$ term in this sequence is the polynomial algebra on generators $h_{1,1}, h_{1,2}, h_{1,4}, h_{1,8}, h_{2,1}, h_{2,2}, h_{2,4}, h_{3,1}, h_{3,2}, h_{4,1}$ from 6.1 , and from 6.32 we find

$$
\begin{aligned}
\delta h_{2,1} & =h_{1,1} h_{1,2}, \\
\delta h_{2,2} & =h_{1,2} h_{1,4}, \\
\delta h_{2,4} & =h_{1,4} h_{1,8}, \\
\delta h_{3,1} & =h_{1,1} h_{2,2}+h_{1,4} h_{2,1}, \\
\delta h_{4,1} & =h_{1,1} h_{3,2}+h_{2,1} h_{2,4}+h_{1,8} h_{3,1} .
\end{aligned}
$$

Thus we have

\subsection{THEOREM.}

$$
E_{2}=P\left(h_{0}, h_{1}, h_{2}, h_{3}, b_{2,1}, b_{2,2}, b_{2,4}, b_{3,1}, b_{3,2}, b_{4,1}, u, r\right) / R
$$


where $R$ is the set of relations

$$
\begin{gathered}
h_{i} h_{i+1}=0, \quad h_{3}^{2} b_{3,1}+b_{2,1} b_{2,4}+h_{0}^{2} b_{3,2}=0, \quad h_{0} u=h_{2} b_{2,1}, \quad h_{2} u=h_{0} b_{2,2}, \\
h_{3} r=h_{1} b_{2,4}, \quad h_{1} r=h_{3} b_{2,2}, \quad h_{3} u=h_{0} r=0, \quad u^{2}=h_{1}^{2} b_{3,1}+b_{2,1} b_{2,2}, \\
r^{2}=h_{2}^{2} b_{3,2}+b_{2,2} b_{2,4}, \quad b_{2,4} u=h_{0} h_{2} b_{3,2}, \quad b_{2,1} r=h_{1} h_{3} b_{3,1} .
\end{gathered}
$$

7.2. From 6.33 we find easily

$$
\begin{aligned}
\delta^{2} b_{2,1} & =h_{0}^{2} h_{2}+h_{1}^{3}, \\
\delta^{2} b_{2,2} & =h_{1}^{2} h_{3}+h_{2}^{3}, \\
\delta^{2} b_{2,4} & =h_{3}^{3}, \\
\delta^{2} b_{3,1} & =h_{1} b_{2,2}+h_{3} b_{2,1}, \\
\delta^{2} b_{3,2} & =h_{2} b_{2,4}, \\
\delta^{2} b_{4,1} & =h_{1} b_{3,2}, \\
\delta^{2} u & =h_{0} h_{2}^{2}, \\
\delta^{2} r & =h_{1} h_{3}^{2}
\end{aligned}
$$

and this gives all the differentials in $E_{2}$. Calculating out this gives

7.3 THEOREM. As an algebra $E_{3}$ has a basis consisting of 31 generators:

$$
\begin{gathered}
h_{0}, h_{1}, h_{2}, h_{3}, b_{2,1}^{2}, b_{2,2}^{2}, b_{2,4}^{2}, b_{3,1}^{2}, b_{3,2}^{2}, b_{4,1}^{2}, \quad c_{0}=\left\{h_{1} u\right\}, \quad c_{1}=\left\{h_{2} r\right\}, \\
d_{0}=\left\{u^{2}\right\}, \quad d_{1}=\left\{r^{2}\right\}, \quad e_{0}=\left\{b_{22} u\right\}, \quad e_{1}=\left\{b_{2,4} r\right\}, \quad K=h_{2} b_{31}, \\
n=\left\{r h_{2} b_{31}\right\}, \quad W=\left\{b_{21} u\left(h_{2} b_{31}\right)\right\}, \quad X=\left\{h_{3} b_{32}\right\}, \quad V=\left\{b_{21} b_{3,2}+h_{1}^{2} b_{41}\right\}, \\
\lambda=\left\{u b_{3,2}\right\}, \quad \alpha=\left\{h_{0} b_{22} b_{31} b_{32}\right\}, \quad \tau=\left\{h_{2} b_{4,1}\right\}, \quad \theta=\left\{h_{0} b_{4,1}\right\}, \\
s=\left\{h_{3}^{2} b_{24} M+h_{0}^{2} b_{24} b_{31}^{2}\right\}, \quad t=\left\{b_{22}^{2} b_{2,1} M\right\}, \quad y=\left\{b_{21}^{2} b_{2,2} M\right\}, \\
z=\{u M\}, \quad a=\left\{h_{2} b_{4,1} b_{3,1}\right\}, \quad a^{\prime}=\left\{b_{21} u h_{2} b_{31} b_{4,1}\right\}
\end{gathered}
$$

where $M=\left(b_{31} b_{32}+b_{22} b_{41}\right)$.

The reader may work out the enormous number of relations between them if he wishes. This is made somewhat easier by referring to the graphs of 7.4.

(The calculation is direct, but as an aid it is convenient to construct a spectral sequence starting from an associated graded version of $E_{2}$ and converging to $E_{3}$, by using the fact that $E_{2}$ is a differential algebra and taking the augmentation filtration [7].)

7.4. To aid the reader we graph some of the parts of $E_{3}$ (with some extensions). We first graph the subalgebra with generators $h_{0}, h_{1}, h_{2}, h_{3}, b_{21}^{2}, b_{22}^{2}, b_{24}^{2}, c_{0}, d_{0}, e_{0}$, $c_{1}, d_{1}, e_{1}, K, W$, (Figure 7.41). Note that in 7.41 we give no products with $b_{31}^{2}$, 
but the product of every element present with $b_{31}^{2}$ is nonzero and independent. Note also that vertical lines connecting two points denote multiplication by $h_{0}$, while a $45^{\circ}$ line is multiplication by $h_{1}$ and a broken line denotes multiplication by $h_{2}$. Finally, the horizontal coordinates are $t-s(t$ is the "internal degree" and $s$ the "simplicial degree") while the vertical coordinates are $s$.

The second graph gives the various multiples by elements in the first graph of the generators $X, V, \lambda, \tau, \theta$. In particular, this gives the $E_{2}$ term of our spectral sequence completely for $t-s \leqq 42$. For completeness in specifying these elements it is also necessary to include $a, a^{\prime}, \alpha, z, t, y$. Thus the family not graphed is generated by $s$, and $b_{3,2}^{2}, b_{2,4}^{2}$. Moreover,

$$
E_{2} \cong P\left(b_{3,1}^{2}, b_{41}^{2}\right) \otimes C
$$

where $C$ is composed of 7.41, 7.42 together with the part given by the five remaining generators above. Finally, we should point out that the products $X V, V \lambda$ etc. do not appear in 7.41, but for completeness we have included $n, d_{1}, e_{1},\left(b_{24}\right)^{2}$ in the graph.

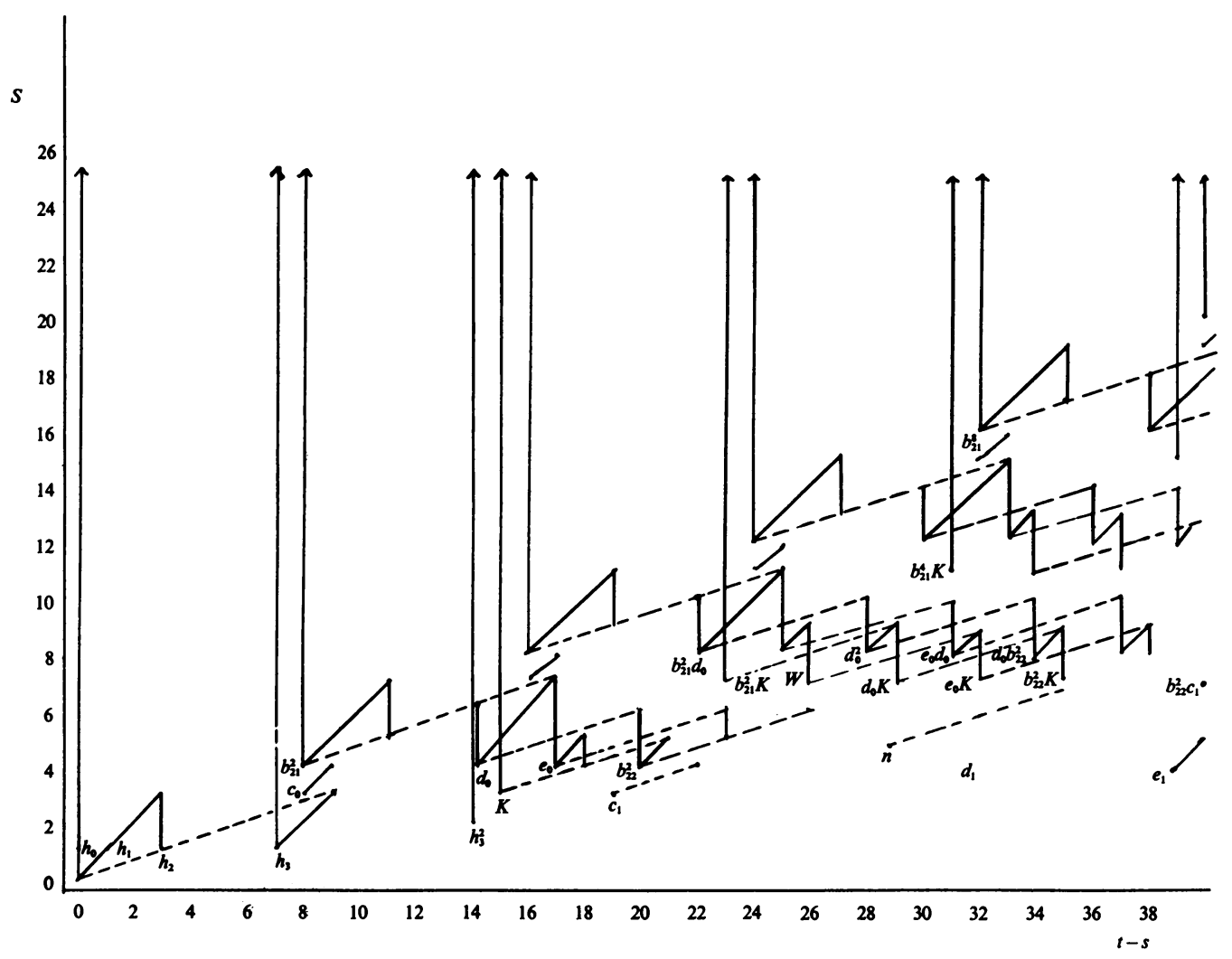

FIGURE 7.41 


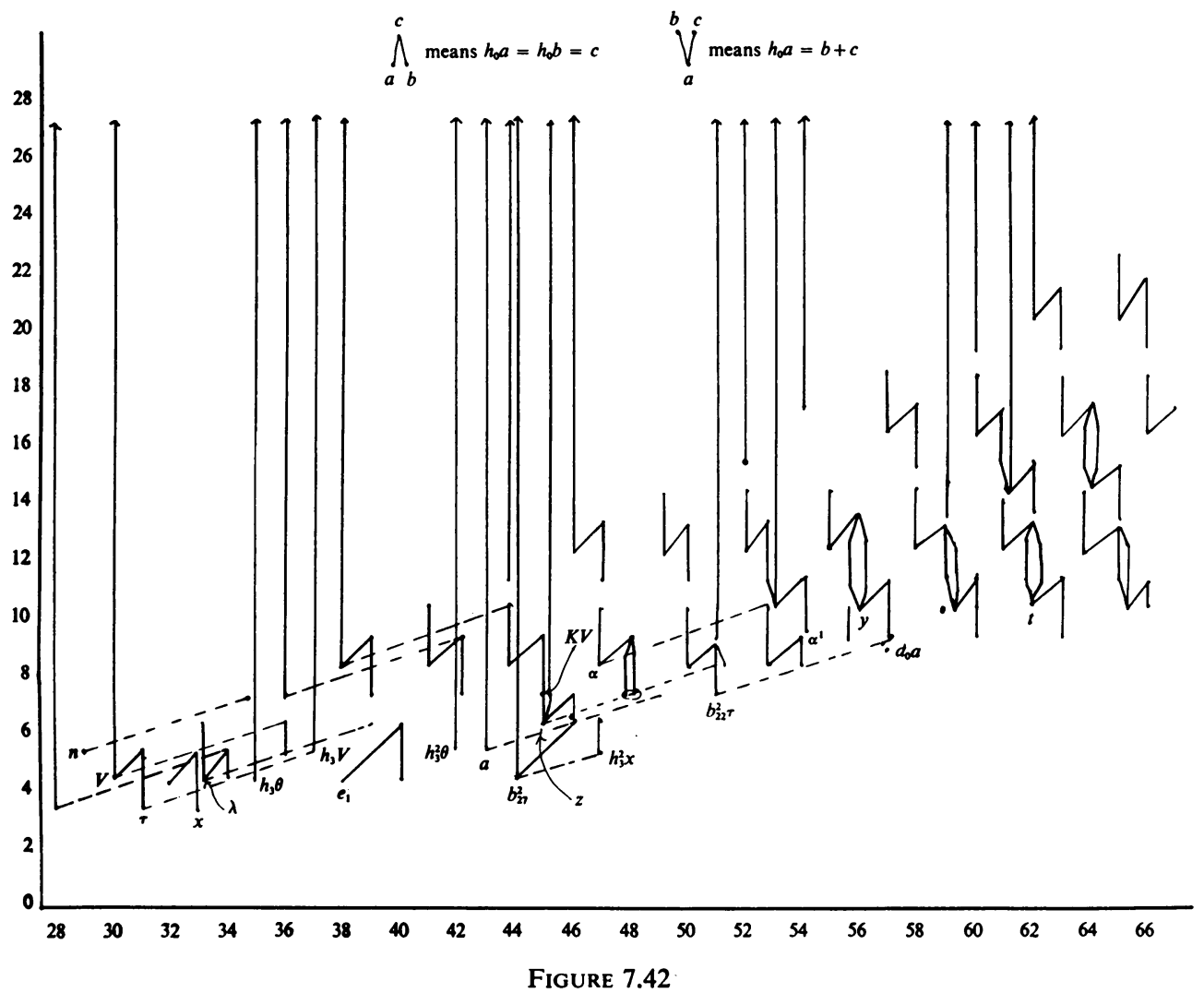

8. The $\delta^{4}$ and $\delta^{6}$ differentials and $\operatorname{Ext}_{\mathscr{A}_{(3)}}\left(Z_{2}, Z_{2}\right)$ through the 42 stem.

8.1. By examination we find $E^{3}=E^{4}$. However, in $E^{4}$ we have

8.11. THEOREM.

(i) $\delta^{4} b_{21}^{2}=h_{0}^{4} h_{3}$,

(ii) $\delta^{4} K=h_{0}^{2} h_{3}$,

(iii) $\delta^{4} a=h_{0} h_{3}^{2} \theta$,

(iv) $\delta^{4} b_{3,1}^{2}=h_{2}\left(b_{2,2}\right)^{2}$,

(v) $\delta^{4} b_{3,2}^{2}=h_{3}\left(b_{2,4}\right)^{2}$,

(vi) $\delta^{4} b_{4,1}^{2}=h_{2}\left(b_{3,2}\right)^{2}$

and $\delta^{4}$ is zero on all other generators.

Proof. It is easy to check that the $\delta^{4}$ differential must be zero on all other generators for filtration reasons. It remains to verify the remaining differentials. We use the notation of 3.6.

(i) Let $L=k_{3} k_{0}^{4}$ in our chain complex. We claim its boundary is $k_{3,1} k_{2,1} k_{0}^{2}+k_{2,1}^{4}$.

(1) $x_{2}\left(k_{3} k_{0}^{i}\right)=S q^{2} k_{3,1} k_{0}^{i-1}+S q^{2} S q^{4} k_{2,1} k_{0}^{i-1}$, thus $\left\{\left[x_{1} x_{2}\right]+x_{1}^{2}\right\} k_{3} k_{0}^{i}=q_{2,1} k_{3,1} k_{0}^{i-2}$ $+\left(q_{3,1}+S q^{4} q_{2,1}\right) k_{2,1} k_{0}^{i-2}$ and 
(2) $x_{3}\left(k_{3} k_{0}^{i}\right)=k_{3,1} k_{2,1} k_{0}^{i-2}+S q^{4} k_{2,1}^{2} k_{0}^{i-2}$ hence $\left\{\left[x_{1}, x_{3}\right]+\left[x_{1}, x_{2}\right]+x_{2}^{2}+x_{1}^{2}\right\}\left(k_{3} k_{0}^{i}\right)$ $=S q^{2} q_{2,1}^{2} k_{0}^{i-3}$ so

(3) $x_{4}\left(k_{3} k_{0}^{i}\right)=S q^{2} k_{2,1}^{3} k_{0}^{i-3}$ implies $\left\{\left[x_{4}, x_{1}\right]+\left[x_{3}, x_{2}\right]+\left[x_{3}, x_{1}\right]+x_{2}^{2}+\left[x_{2}, x_{1}\right]+x_{1}^{2}\right\}$ $\times\left(k_{3} k_{0}^{i}\right)=k_{2,1}^{4} k_{0}^{i-4}$ and here the process stops and (i) follows.

(ii) Let $K(j)=k_{3}^{2} k_{0}^{j}$ then

$$
\begin{aligned}
\boldsymbol{x}_{2}(K(j))= & S q^{4} k_{2,4} k_{0}^{j}+S q^{7} q_{2,1} k_{2,2} k_{0}^{j} \\
& +\left(S q^{3}+q_{2,1}\right) q_{2,2} k_{3,1} k_{0}^{j}+S q^{2} k_{3,1} k_{3} k_{0}^{j-1}+S q^{2} S q^{4} k_{2,1} k_{3} k_{0}^{j-1}
\end{aligned}
$$

and (ignoring terms on which $S^{\otimes}$ will be zero).

$$
\begin{aligned}
\left\{x_{1}^{2}+\left[x_{1}, x_{2}\right]\right\} K(j)= & S q^{1} q_{2,1} q_{3,1} k_{2,2} k_{0}^{j-1}+\left(S q^{2}+q_{2,1}\right) q_{3,1} k_{3,1} k_{0}^{j-1} \\
& +q_{2,1} q_{3,1} k_{3,1} k_{0}^{j-1}+\left(S q^{1} q_{2,2} q_{3,1}+S q^{2} q_{2,4}\right) k_{2,1} k_{0}^{j-1} \\
& +\left(S q^{4} q_{2,1}+q_{3,1}\right) k_{2,1} k_{3} k_{0}^{j-2}
\end{aligned}
$$

thus

$$
\begin{aligned}
\boldsymbol{x}_{3}(K(j))= & S q^{1} q_{2,1} k_{3,1} k_{2,2} k_{0}^{j-1}+S q^{3} k_{3,1}^{2} k_{0}^{j-1} \\
& +S q^{1} q_{2,2} k_{3,1} k_{2,1} k_{0}^{j-1}+S q^{4} k_{2,1}^{2} k_{3} k_{0}^{j-2}+k_{3,1} k_{2,1} k_{3} k_{0}^{j-2}, \\
x_{4}(K(j))= & S q^{1} k_{3,1}^{2} k_{2,2} k_{0}^{j-2}+k_{2,4} k_{2,1}^{2} k_{0}^{j-2} \\
& +S q^{3} q_{2,1} k_{2,2} k_{2,1}^{2} k_{0}^{j-2}+S q^{2} k_{2,1}^{3} k_{3} k_{0}^{j-3} .
\end{aligned}
$$

Hence the reduced boundary of $k_{3}^{2} k_{0}^{2}$ is $k_{2,4} k_{2,1}^{2}+k_{3,1} k_{2,1} k_{3}$. On the other hand,

$$
\left(x_{2}+x_{3}\right) k_{21}^{2} k_{3} k_{2}=k_{24} k_{2,1}^{2}+k_{3,1} k_{2,1} k_{3}+k_{3,1}^{2} k_{2}
$$

and (ii) follows.

(iii) $h_{0} a=K \theta$, hence $\delta^{4} K \theta=h_{0}^{2} h_{3}^{2} \theta$ so $\delta^{4}(a)=h_{0} h_{3}^{2} \theta+v$ and by observation $v=0$.

(iv) $\left[S q^{4}, q_{2,2}\right]=\left(q_{2}+S q^{3}\right) q_{3,1}\left[S q^{3}, q_{22}\right]=S q^{2} q_{3,1}\left[S q^{2}, q_{2,2}\right]=S q^{1} q_{3,1}\left[S q^{1}, q_{22}\right]=$ $q_{3,1}$ thus $x_{4}\left(k_{2,2}^{(4)} k_{2}\right)=k_{3,1}^{(4)}$ and this proves (iv). The remaining cases are similar.

8.2. After calculating $E_{5}$ through $t-s<42$ we find the only possible remaining differential is a $\delta^{(6)}$. Specifically $\delta^{(6)}\left(b_{2,1}^{4} K\right)=u h_{0}^{8} V$ where $u=0$ or 1 . In fact we have

8.21 Proposition. (a) $h_{3}\left(b_{21}^{2} K\right)=h_{0}^{4} V$ and (b) $\delta^{(6)}\left(b_{21}^{4} K\right)=h_{0}^{8} V$.

8.22 Remark. 8.21. (a) was first observed by M. Mahowald using very sophisticated methods. It implies (b) since $h_{0}^{4} h_{3}=0$ and the only thing which can hit $h_{0}^{8} V$ is $\left(b_{2,1}^{4} K\right)$. We prove 8.21 using only the theory developed here.

Proof. We build up cycles dual to $V, \ldots, h_{0}^{4} V$,

(0) $k_{3,2}^{2} k_{2,1}^{2}$ is a cycle dual to $V$.

(1) $\partial k_{3,2}^{2} k_{2,1}^{2} k_{0}^{i}=k_{4,1} k_{3,2} k_{2,1}^{2} k_{0}^{i-1}$ so $k_{3,2}^{2} k_{2,1}^{2} k_{0}+k_{4,1} k_{2,4} k_{2,1}^{2} k_{1}$ represents a cycle dual to $h_{0} V$.

(2) $k_{4,1} k_{2,4} k_{2,1}^{2} k_{1} k_{0}^{i}$ adds error $k_{41} k_{24} k_{21}^{3} k_{0}^{i-1}$ so $k_{3,2}^{2} k_{2,1}^{2} k_{0}^{2}+k_{4,1} k_{2,4} k_{2,1}^{2} k_{1} k_{0}$ $+k_{2,4}^{2} k_{2,1}^{4}$ represents $\left(h_{0}^{2} V\right)^{*}$.

(3) $k_{4,1} k_{2,4} k_{2,1}^{2} k_{1} k_{0}^{i}$ now adds a further error, namely $k_{4,1} k_{3,1}^{2} k_{21}^{2} k_{1} k_{0}^{i-2}$. To correct this we add 
$k_{3,1}^{3} k_{2,1}^{2} k_{3} k_{1}$ (note here that though $k_{21}^{2} k_{3}$ contributes a term $k_{31}^{2}$ since $\left(\begin{array}{l}5 \\ 2\end{array}\right)=0(2)$ this term does not appear).

(4) $k_{3,1}^{3} k_{2,1}^{2} k_{3} k_{1} k_{0}$ adds error $k_{31}^{3} k_{21}^{3} k_{3}$ while $k_{2,4}^{2} k_{2,1}^{4} k_{0}^{2}$ adds $k_{3,1}^{2} k_{2,4} k_{21}^{4}$. Thus we add $k_{31}^{2} k_{2,1}^{4} k_{3} k_{2}$ which adds error $k_{4,1} k_{31} k_{21}^{4} k_{2}$. We correct this in turn with $k_{31} k_{24} k_{2,1}^{5} k_{2}$. Note that the term $k_{3,1}^{3} k_{2,1}^{2} k_{3} k_{1} k_{0}+k_{3,1}^{2} k_{2,1}^{4} k_{3} k_{2}$ in $\left(h_{0}^{4} V\right)^{*}$ shows $h_{3}\left(b_{21}^{2} K\right)=h_{0}^{4} V+\lambda$. However, there is only $h_{0}^{4} V$ in $(38,8)$ and $8.21(\mathrm{a})$ follows. Thus as noted in 8.22 the proof is complete.

8.4. Now using $7.41,7.42$ it is a routine matter to calculate the $E_{\infty}$ term of the spectral sequence for $t-s<41$ and we tabulate the result in Figure 8.41 (actually we have done all the work to determine $\operatorname{Ext}_{\mathscr{A}_{3}}^{t, s}\left(Z_{2}, Z_{2}\right)$ for $t-s$ much larger than 40 but we leave this to the reader).

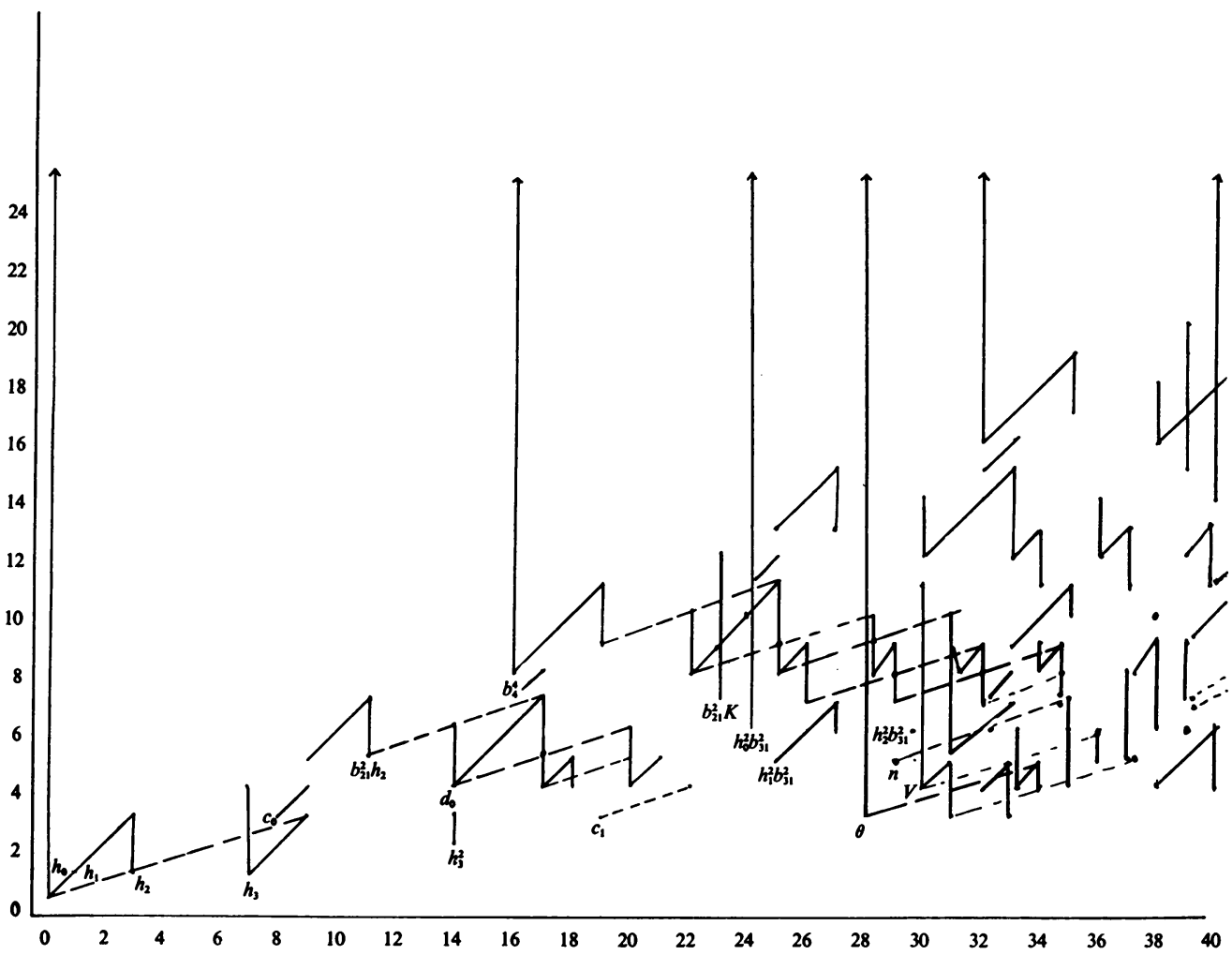

FIGURE 8.41

\section{REFERENCES}

1. V. K. A. M. Gugenheim, On twisted tensor products of algebras (mimeographed), Johns Hopkins Univ., Baltimore, Md., 1960.

2. - On extensions of algebras, co-algebras and Hopf algebras. I, Amer. J. Math. 84 (1962), 349-382. MR 26 \#1340. 
3. Arunas Liulevicius, Multicomplexes and a general change of rings theorem (mimeographed, Chicago, 1967).

4. Saunders Mac Lane, Homology, Die Grundlehren der math. Wissenschaften, Band 114, Academic Press, New York and Springer-Verlag, Berlin, 1963. MR 28 \#122.

5. J. F. Adams, On the non-existence of elements of Hopf invariant one, Ann. of Math. (2) 72 (1960), 20-104. MR $25 \# 4530$.

6. J. Milnor and J. C. Moore, On the structure of Hopf algebras, Ann. of Math. (2) 81 (1965), 211-264. MR 30 \#4259.

7. J. P. May, Thesis, Princeton Univ., Princeton, N. J., 1964.

8. H. Cartan, Sur l'itération des opérations de Steenrod, Comment Math. Helv. 29 (1955), 40-58. MR 16, 847.

9. N. E. Steenrod and D. B. A. Epstein, Cohomology operations, Ann. of Math. Studies, no. 50, Princeton Univ. Press, Princeton, N. J., 1962. MR 26 \#3056.

10. J. Milnor, The Steenrod algebras and its dual, Ann. of Math. (2) 67 (1958), 150-171. MR 20 \#6092.

University of Illinois at Chicago Circle, Chicago, Illinois 60680 